\title{
Burden of illness in infants and young children hospitalized for respiratory syncytial virus: $A$ rapid review
}

\author{
Aireen Wingert ${ }^{1 *}$, Jennifer Pillay ${ }^{1}$, Dorothy L Moore ${ }^{2}$, Samantha Guitard ${ }^{1}$, Ben Vandermeer ${ }^{1}$, \\ Michele P Dyson ${ }^{1}$, Angela Sinilaite ${ }^{2}$, Matthew Tunis ${ }^{2}$, Lisa Hartling ${ }^{1}$
}

\begin{abstract}
Respiratory syncytial virus (RSV) infections are common among young children and represent a significant burden to patients, their families and the Canadian health system. Here we conduct a rapid review of the burden of RSV illness in children 24 months of age or younger. Four databases (Medline, Embase, Cochrane Database of Clinical Trials, ClinicalTrials.gov from 2014 to 2018), grey literature and reference lists were reviewed for studies on the following: children with or without a risk factor, without prophylaxis and with lab-confirmed RSV infection. Of 29 studies identified, 10 provided within-study comparisons and few examined clinical conditions besides prematurity. For infants of 33-36 weeks gestation (wGA) versus term infants, there was low-to-moderate certainty evidence for an increase in RSV-hospitalizations ( $n=599,535$ infants; RR 2.05 [95\% Cl 1.89-2.22]; 1.3 more per 100 [1.1-1.5 more]) and hospital length of stay ( $n=7,597$ infants; mean difference 1.00 day [ $95 \% \mathrm{Cl} 0.88-1.12])$. There was low-to-moderate certainty evidence of little-to-no difference for infants born at 29-32 versus 33-36 wGA for hospitalization ( $n=12,812$ infants; RR 1.20 [95\% Cl 0.92-1.56]). There was low certainty evidence of increased mechanical ventilation for hospitalized infants born at $29-32$ versus $33-35$ wGA ( $n=212$ infants; $R R 1.58,95 \% \mathrm{Cl} 0.94-2.65$ ). Among infants born at 32-35 wGA, hospitalization for RSV in infancy may be associated with increased wheeze and asthma-medication use across six-year follow-up (RR range 1.3-1.7). Children with versus without Down syndrome may have increased hospital length of stay $(n=7,206$ children; mean difference 3.00 days, $95 \% \mathrm{Cl} 1.95-4.05$; low certainty). Evidence for other within-study comparisons was of very low certainty. In summary, prematurity is associated with greater risk for RSV-hospitalization and longer hospital length of stay, and Down syndrome may be associated with longer hospital stay for RSV. Respiratory syncytial virus-hospitalization in infancy may be associated with greater wheeze and asthma-medication use in early childhood. Lack of a comparison group was a major limitation for many studies.
\end{abstract}

This work is licensed under a Creative Commons Attribution 4.0 International License.

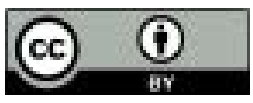

Affiliations

${ }^{1}$ Alberta Research Centre for Health Evidence, Department of Pediatrics, Faculty of Medicine and Dentistry, University of Alberta, Edmonton, $\mathrm{AB}$

2 Public Health Agency of Canada, Ottawa, ON

\section{*Correspondence:}

awingert@ualberta.ca

Suggested citation: Wingert A, Pillay J, Moore DL, Guitard S, Vandermeer B, Dyson MP, Sinilaite A, Tunis M, Hartling L. Burden of illness in infants and young children hospitalized for respiratory syncytial virus: A rapid review. Can Commun Dis Rep 2021;47(9):381-96. https://doi.org/10.14745/ccdr.v47i09a05

Keywords: respiratory syncytial virus, disease burden, hospitalization, systematic review

\section{Introduction}

Respiratory syncytial virus (RSV) infections are common among young children $(1,2)$, presenting as bronchiolitis, pneumonia, or other respiratory morbidity (3). Hospitalization due to RSV is a significant burden for patients, families and the Canadian health system (4).

Increased risk for RSV-hospitalization has been associated with age younger than one year $(3,5)$, prematurity $(6)$, chronic lung disease (7), congenital heart disease (8), other chronic conditions including cystic fibrosis, immunodeficiency (9-12) and residence in Indigenous, northern or remote communities (13). These populations may also have higher rates of admission to intensive care units (ICU), requirements for respiratory support, and higher mortality attributable to RSV (9). RSV-hospitalization in the first two years of life has also been associated with wheezing in childhood (14-16). 
While no active vaccines exist for RSV prophylaxis, the monoclonal antibody palivizumab (Synagis ${ }^{\circledR}$, AstraZeneca) has demonstrated effectiveness in preventing RSV-hospitalization among some high risk populations $(17,18)$. However, while efficacy of palivizumab (PVZ) in clinical trials appears to be high for children with some underlying clinical conditions, real-world evidence from observational studies is less certain (2), with wide variations in effectiveness. Due to the high numbers needed to treat in order to prevent hospitalization and the relatively high cost of PVZ, most jurisdictions use the intervention sparingly for select groups at highest risk of severe disease. Additionally, RSV vaccine development has been well under way, with some vaccine candidates undergoing phase 3 clinical trials (19). There is currently no global consensus on RSV risk groups and variable policies exist even within Canada.

The objective of this rapid review is to address the following question: What is the burden of RSV illness including long-term sequelae among children 24 months of age and younger without prophylaxis, and with or without risk factors for severe RSV disease, and for immunocompromised children younger than 18 years of age?

Findings from the review will help inform updated recommendations of Canada's National Advisory Committee on Immunization on the use of PVZ prophylaxis to prevent severe consequences of RSV infection. This evidence base will also be relevant for future deliberations on program design for anticipated RSV vaccines and newer monoclonal antibodies (19).

\section{Methods}

This review was guided by methods for reviews of interventions (20), overall prognosis (21), and risk of future event (prognosis) (22); a protocol was developed a priori (Supplement 1), and reviewed and approved by the National Advisory Committee on Immunization RSV Working Group. In light of the restricted literature search timeframe of interest to the review commissioners, we refer to the undertaken work as a rapid review.

\section{Literature search}

Searches were conducted on September 6, 2018, in Medline, Embase, Cochrane Database of Clinical Trials, ClinicalTrials. gov, and websites of international public health authorities (Supplement 2). Limits were applied for date of publication (January 1, 2014 to September 6, 2018) and language (English or French). The date limit was aimed at capturing outcomes just before and after significant changes in clinical practice stemming from the revised recommendations for PVZ prophylaxis by the American Academy of Pediatrics (23) as well as the Canadian Paediatric Society (2).

\section{Study selection and eligibility criteria}

Two reviewers independently screened titles and abstracts followed by full texts. Discrepancies were resolved by discussions.

Studies conducted in Organisation for Economic Co-operation and Development (OECD) countries, including observational studies and placebo groups of controlled trials were eligible for inclusion. Studies reporting on children 24 months of age and younger, with or without a risk factor of interest, or immunocompromised children 18 years of age and younger without PVZ prophylaxis and with lab-confirmed RSV infection were eligible. Children without RSV infection were eligible as a comparator group for long-term outcomes. Short-term outcomes included RSV-hospitalization, hospital length of stay, ICU admission and length of stay, oxygen support and duration, mechanical ventilation and duration, extracorporeal membrane oxygenation and duration, case fatality (death due to RSV), and complications from RSV infection (e.g. secondary infection). Long-term outcomes (minimum one-year follow-up) included self-reported, parent-reported or physician-diagnosed recurrent wheeze, atopic asthma, deterioration of pulmonary or cardiac function, and impaired growth or development. Detailed inclusion and exclusion criteria are in Supplement 3.

\section{Data extraction, synthesis/analysis and risk of bias assessment}

One reviewer extracted data with second-reviewer verification.

For dichotomous outcomes, we extracted the number of events and the number analysed in each eligible group, or relative measures (e.g. odds ratio) if crude events were not reported. For continuous outcomes, mean values for each time-point, and change scores, including standard deviations or measures of variability were extracted. Risk ratio (RR) with $95 \%$ confidence interval $(\mathrm{Cl})$ and mean difference (MD) were used for comparisons between groups.

Our primary interest was using data from studies that reported on two or more groups, either having different risk factors, or a risk group versus healthy term infants (within-study/direct comparisons). For similar comparisons reported by more than one study, data were pooled using the DerSimonian Laird random effects model inverse variance method with Mantel-Haenszel weighting. Risk differences were used when rare or zero events appeared in at least one study group. We also made comparisons between short-term outcomes in risk groups and healthy term infants reported by different studies (between-study/indirect comparisons). We used the double-arc sine transformation to pool single-group proportions across multiple studies. When no comparison was made, we report event proportions for the single group in these studies.

For outcomes where estimates were statistically significant, we calculated the absolute risk difference (24). 
Analyses were performed using Excel, Review Manager (version 5.3) and STATA (version 14.2).

Two reviewers independently assessed the risk of bias for each study, using a modified tool based on the Quality Assessment Tool for Observational Cohort and Cross-sectional Studies and the Quality In Prognosis Studies (QUIPS) tool (Supplement 4). Disagreements were resolved via consensus or third-reviewer consultation.

\section{Certainty of evidence}

Two reviewers independently assessed the certainty of evidence for each outcome (as high, moderate, low, or very low) from within-study comparisons (direct evidence), with disagreements resolved through consensus. The approach followed principles of the Grading of Recommendations Assessment, Development and Evaluation working group and considerations for a body of evidence that examines risk of future events (prognosis)

(Supplement 5) (21)

\section{Results}

\section{Study selection and characteristics}

Twenty-nine cohort studies were included (Figure 1, Table 1, and Supplements 6 and 7) (13,25-52); of these, 10 reported at least one within-study comparison $(26-28,31,32,36,37,42,50,52)$. Twelve studies were conducted in the United States $(25,26,33,34,36,38,42,45-47,49,50)$, three each, in Canada $(13,29,48)$ and the Netherlands $(30,43,52)$, two each, in
Figure 1: Flow diagram of study selection

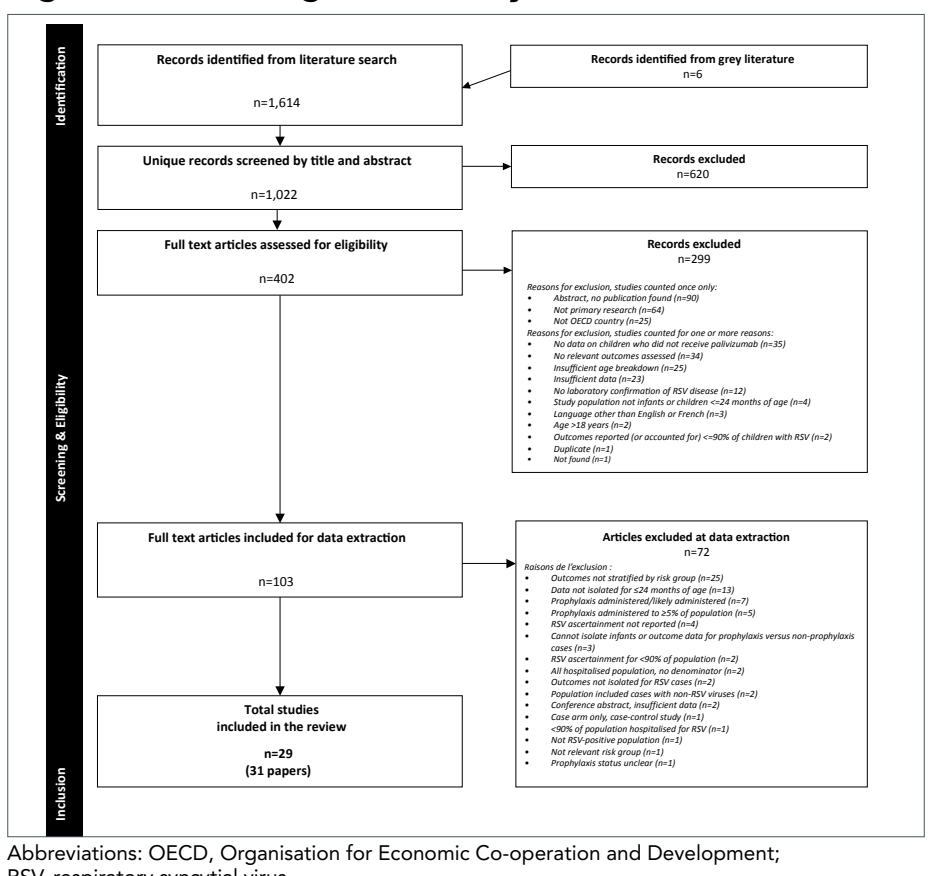
RSV, respiratory syncytial virus

Finland $(27,28)$, France $(35,37)$ and Japan $(40,41)$ and one each in Chile (44), Denmark (31), Ireland (39), Spain (32) and multiple countries (51). Thirteen $(13,25,26,29-32,37,43,45,46,51,52)$ studies had some form of industry funding. Three papers reported on the same study: primary publication by Ambrose et al. (25), with associated publications by Franklin et al. (53) and Simões et al. (54).

\section{Table 1: Summary of included studies}

\begin{tabular}{|c|c|c|c|c|c|}
\hline $\begin{array}{l}\text { Study design \& } \\
\text { setting } \\
\text { (no. of studies) }\end{array}$ & $\begin{array}{c}\text { Risk groups } \\
\text { (no. of studies }{ }^{a} \text { ) }\end{array}$ & $\begin{array}{l}\text { RSV infection } \\
\text { (no. of studies) }\end{array}$ & 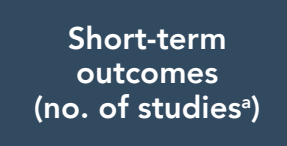 & $\begin{array}{l}\text { Long-term } \\
\text { outcomes and } \\
\text { follow-up } \\
\text { (no. of studies } \text { ) }\end{array}$ & $\begin{array}{l}\text { Risk of bias by } \\
\text { outcome } \\
\text { (no. of studies }^{a} \text { ) }\end{array}$ \\
\hline $\begin{array}{l}\text { Study design: } \\
\text { - } \text { Prospective cohort } \\
\text { - }(n=15) \\
\text { Retrospective cohort } \\
(n=12) \\
\text { - Retrospective follow- } \\
\text { up of prospective } \\
\text { cohort ( } n=1) \\
\text { - } \text { Retrospective cohort } \\
\text { with non-concurrent } \\
\text { control ( }(n=1) \\
\text { Country: } \\
\text { - United States ( } n=12) \\
\text { - Canada }(n=3) \\
\text { - Netherlands }(n=3) \\
\text { - Finland }(n=2) \\
\text { - Japan }(n=2) \\
\text { - France }(n=2) \\
\text { - Chile }(n=1) \\
\text { - Denmark }(n=1) \\
\text { - } \text { Ireland }(n=1) \\
\text { - Spain }(n=1) \\
\text { - International, } \\
\text { multi-site }(n=1)\end{array}$ & 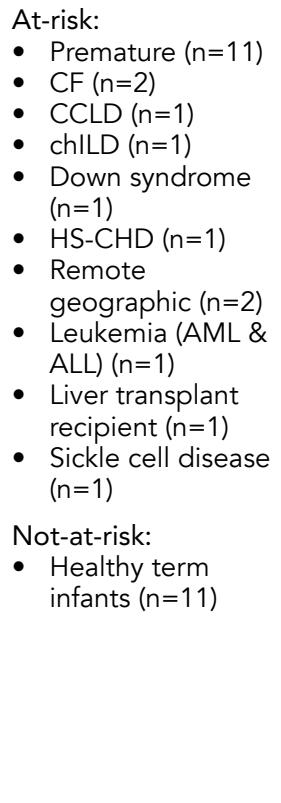 & 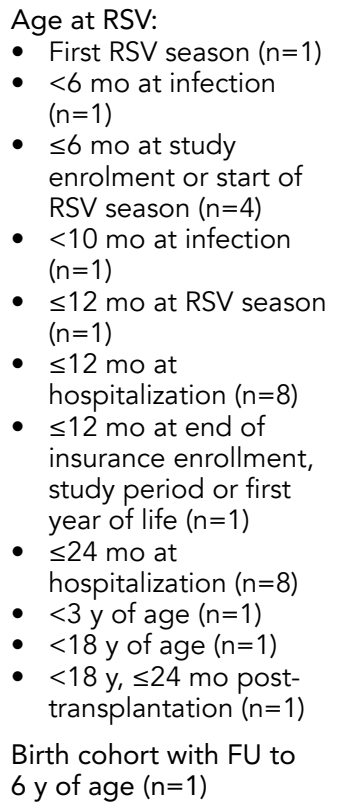 & $\begin{array}{l}\text { Incidence of } \\
\text { RSV-hospitalization } \\
(n=23) \\
\text { Hospital LOS }(n=16) \\
\text { ICU admission }(n=13) \\
\text { ICU LOS ( } n=5) \\
\text { Oxygen therapy } \\
\text { ( } n=6) \\
\text { Oxygen therapy } \\
\text { duration }(n=5) \\
\text { MV ( } n=15) \\
\text { MV duration }(n=4) \\
\text { Case fatality }(n=7)\end{array}$ & $\begin{array}{l}\text { Wheeze: } \\
\text { - At } 1 \text { y FU }(n=1) \\
\text { - Across } 2-6 \text { y of } \\
\text { age }(n=1) \\
\text { - At } 6 \text { y of age }(n=1) \\
\text { Asthma: } \\
\text { - Across } 2-6 \text { y of } \\
\text { age }(n=1) \\
\text { - At } 7 \text { y of age }(n=1) \\
\text { - At } 17-20 \text { y of age } \\
\quad(n=1) \\
\text { - At } 28-31 \text { y of age } \\
\quad(n=1) \\
\text { Lung function: } \\
\text { - At } 6 \text { y of age }(n=1) \\
\text { - At } 17-20 \text { y of age } \\
(n=1) \\
\text { - At } 28-31 \text { y of age } \\
(n=1)\end{array}$ & $\begin{array}{l}\text { Incidence of } \\
\text { RSV-hospitalization: } \\
\text { - High }(n=10) \\
\text { - Moderate }(n=11) \\
\text { Short-term } \\
\text { outcomes: } \\
\text { - High }(n=2) \\
\text { - Moderate }(n=9) \\
\text { - Low }(n=10) \\
\text { Long-term } \\
\text { outcomes: } \\
\text { - Moderate }(n=5) \\
\text { - Low }(n=1)\end{array}$ \\
\hline
\end{tabular}

Abbreviations: ALL, acute lymphoblastic leukemia; AML, acute myeloid leukemia; CCLD, congenital cystic lung disease; CF, cystic fibrosis; chILD, childhood interstitial lung disease; FU, follow-up;

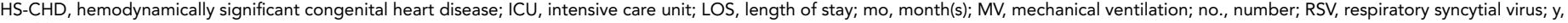
year(s)

a Study may contribute to more than one risk group, outcome and/or follow-up duration 
Eighteen studies $(25,26,29,30,33-37,39-43,45,48,50,51)$ included children either not given or not considered for PVZ prophylaxis prior to RSV-hospitalization; four studies $(13,32,46,47)$ reported prophylaxis among less than $5 \%$ of the applicable population with RSV, and seven studies $(27,28,31,38,44,49,52)$ were considered by clinical judgement to not have included children who received prophylaxis. One study included some children who may have received prophylaxis (34).

We included three studies of children with RSV older than 24 months of age to capture evidence for immunocompromised populations: children three years old or younger with Down syndrome with or without known risk factors for RSV (50), and younger than 18 years old with liver transplantation (38) and sickle cell disease (49).

Studies of at-risk populations reporting short-term outcomes included the following: infants with premature birth (eleven studies) $(25,26,30,32,36,37,42,43,47,48,51)$; cystic fibrosis (two studies) $(29,39)$; one study each for congenital cystic lung disease (40), childhood interstitial lung disease (35), Down syndrome (50), sickle cell disease (49), acute leukemia (41) and prior liver transplant (38); and children residing in remote geographic locations (two studies) $(13,46)$.

Seven studies reporting short-term outcomes included data on healthy term infants hospitalized for RSV $(33,37,42,44,45,50,52)$.

Six studies reported on long-term outcomes: healthy term infants with versus without RSV in infancy $(27,28,31,52)$, premature infants with versus without RSV-hospitalization in infancy (32), and premature versus term infants hospitalized for RSV in their first RSV season (37).

\section{Risk of bias}

Risk of bias ratings are in Table 1 and Supplements 4 and 6 . Studies that reported incidence of RSV-hospitalization were at moderate-to-high risk of bias, mainly due to lack of blinding to childrens' risk status by healthcare providers that may have influenced admission to hospital. For other short-term outcomes, studies were mostly at moderate risk of bias $(25,26,29,33,35,39,45-47)$. Two studies were at high risk of bias due to concerns in more than one domain $(41,44)$. Nearly all reported long-term outcomes $(27,28,32,37,52)$ were at moderate risk of bias, arising from lack of blinding for patient or parent-reported outcomes and/or potential selection biases.

\section{Short-term outcomes from within-study comparisons}

Table 2 summarizes evidence for short-term outcomes from within-study comparisons. Here we do not report further on findings having very low certainty of evidence.
Different degrees of prematurity: One study found little-to-no difference in RSV-hospitalization during their first RSV season for infants born at 29-32 compared with 33-36 weeks' gestation (wGA) (36). This study found little-to-no difference for hospital stay of less than one day versus one day or more between infants born at 29-32 or 33-36 wGA (36).

Another study of infants born at 29-32 versus 33-35 wGA who were hospitalized for RSV in the first year of life found littleto-no difference for ICU admission, but longer (although not statistically significant) ICU length of stay among the infants born at 29-32 wGA (26). There was a greater need for mechanical ventilation for hospitalized infants born at 29-32 wGA versus 33-35 wGA (26).

There were no studies of premature infants born before 29 wGA.

Premature versus term infants: One study found that being born late-premature (33-36 wGA) versus at term was associated with increased RSV-hospitalization in the first two years of life (42). The same study found slightly longer hospitalization for the preterm group (42).

Down syndrome: One study comparing children with Down syndrome without additional risk factors for RSV versus healthy children, all followed to three years of age, reported a higher hospitalization rate in those with Down syndrome (50). There was a discrepancy between the text and tables for RSV-hospitalization rates in these groups that could not be resolved due to unsuccessful attempts to contact the study authors (50). This study also found that RSV was associated with longer hospital length of stay among children with Down syndrome without other risk factors versus children without Down syndrome (50). For all cases of Down syndrome, including those with known risk factors for RSV, the authors conclude that Down syndrome is independently associated, after adjusting for known risk factors for RSV disease, with an increased risk for RSV-hospitalization (50). Of note, data on children younger than 24 months of age without risk factors were not isolated from those with additional risk factors, and therefore, were not used in our analysis.

\section{Select short-term outcome comparisons: Other data}

Supplement 8 contains single risk group data and pooled analyses (when appropriate). Data for between-study comparisons are in Supplement 9. Findings for select outcomes are reported below; data on other short-term outcomes are in Supplement 8.

Single group proportions for RSV-hospitalizations were $5.1 \%$ in the first six months of life and $3.3 \%$ in the first two years of life for infants 29 to younger than 33 wGA $(36,37)$ and $32 / 33$ to 35 
Table 2: Summary of evidence for short-term outcomes among within-study population comparisons

\begin{tabular}{|c|c|c|c|c|c|c|c|c|}
\hline \multirow[b]{2}{*}{ Outcome } & \multirow[b]{2}{*}{$\begin{array}{c}\text { Comparator } \\
1\end{array}$} & \multirow[b]{2}{*}{$\begin{array}{c}\text { Comparator } \\
2\end{array}$} & \multirow{2}{*}{$\begin{array}{c}\text { Study } \\
\text { design } \\
\text { (no. of } \\
\text { studies); } \\
\text { Sample size }\end{array}$} & \multicolumn{2}{|c|}{$\begin{array}{c}\text { Absolute difference } \\
(95 \% \mathrm{Cl})\end{array}$} & \multirow{2}{*}{$\begin{array}{l}\text { Relative } \\
\text { risk } \\
(95 \% \mathrm{Cl})\end{array}$} & \multirow{2}{*}{$\begin{array}{l}\text { Certainty } \\
\text { of } \\
\text { evidence }\end{array}$} & \multirow[b]{2}{*}{ Conclusion } \\
\hline & & & & $\begin{array}{c}\text { Comparator } \\
2 \text { risk }\end{array}$ & $\begin{array}{c}\text { Absolute } \\
\text { risk } \\
\text { difference }^{a}\end{array}$ & & & \\
\hline \multicolumn{9}{|c|}{ RSV-hospitalization } \\
\hline $\begin{array}{l}\text { At-risk } \\
\text { population }\end{array}$ & $\begin{array}{l}\text { Prematurity: } \\
\text { 29-32 wGA }\end{array}$ & $\begin{array}{l}\text { Prematurity: } \\
\text { 33-36 wGA }\end{array}$ & $\begin{array}{l}\mathrm{RC}^{36}(\mathrm{n}=1) \\
12,812\end{array}$ & 4.2 per 100 & NS & $\begin{array}{l}\text { RR 1.20 } \\
(0.92 \\
1.56)\end{array}$ & $\begin{array}{l}\text { Moderate } \\
\text { to low } w^{b, c, d}\end{array}$ & $\begin{array}{l}\text { Little to no difference } \\
\text { For RSV-hospitalization in } \\
\text { their first RSV season among } \\
\text { infants born premature at } \\
29-32 \text { wGA vs. } 33-36 \text { wGA }\end{array}$ \\
\hline $\begin{array}{l}\text { At-risk vs. } \\
\text { not-at-risk } \\
\text { population }\end{array}$ & $\begin{array}{l}\text { Prematurity: } \\
\text { 33-36 wGA }\end{array}$ & $\begin{array}{l}\text { Term: } \geq 37 \\
\text { wGA }\end{array}$ & $\begin{array}{l}\mathrm{RC}^{42}(\mathrm{n}=1) \\
599,535\end{array}$ & 1.2 per 100 & $\begin{array}{l}1.3 \text { more per } \\
100 \\
(1.1 \text { to } 1.5 \\
\text { more) }\end{array}$ & $\begin{array}{l}\text { RR 2.05 } \\
(1.89, \\
2.22)\end{array}$ & $\begin{array}{l}\text { Moderate } \\
\text { to low } w^{b, c, d}\end{array}$ & $\begin{array}{l}\text { Increase } \\
\text { RSV-hospitalization by age } \\
<24 \text { months among infants } \\
\text { born premature ( } 33-36 \text { wGA) } \\
\text { vs. at term } \\
\text { Among this group, infants } \\
\text { born at } 33-34 \text { wGA had } \\
\text { highest incidence density for } \\
\text { RSV hospitalization at } 6-12 \\
\text { months of age } \\
\text { (adjusted hazard ratio [aHR] } \\
1.74 \text { [1.17-2.58], p<0.05) and } \\
12-24 \text { months of age (aHR } \\
1.96 \text { [1.26-3.05], p<0.05) } \\
\text { compared to term infants }\end{array}$ \\
\hline $\begin{array}{l}\text { At-risk vs. } \\
\text { not-at-risk } \\
\text { population }\end{array}$ & $\begin{array}{l}\text { Prematurity: } \\
<33 \text { wGA }\end{array}$ & $\begin{array}{l}\text { Term: 39-41 } \\
\text { wGA }\end{array}$ & $\begin{array}{l}\text { RFUPC } \\
(n=1) ; \\
443\end{array}$ & 1.5 per 100 & $\begin{array}{l}4.3 \text { more per } \\
100 \\
(0.2 \text { to } 18 \\
\text { more })\end{array}$ & $\begin{array}{l}\text { RR } 3.88 \\
(1.13, \\
13.30)\end{array}$ & Very low $w^{b, c, e}$ & $\begin{array}{l}\text { Very uncertain } \\
\text { For RSV-hospitalization in } \\
\text { their first RSV season among } \\
\text { infants born at }<33 \text { wGA vs. } \\
\text { at term }\end{array}$ \\
\hline
\end{tabular}

Hospital length of stay, mean days

\begin{tabular}{|c|c|c|c|c|c|c|c|}
\hline $\begin{array}{l}\text { At-risk } \\
\text { population }\end{array}$ & $\begin{array}{l}\text { Prematurity: } \\
29-32 \text { wGA }\end{array}$ & $\begin{array}{l}\text { Prematurity: } \\
\text { 33-35 wGA }\end{array}$ & $\begin{array}{l}\mathrm{PC}^{26}(\mathrm{n}=1) \\
212\end{array}$ & $\begin{array}{l}\text { MD } 4.00 \\
(1.54,6.46)\end{array}$ & N/A & Very lowb,cee & $\begin{array}{l}\text { Very uncertain } \\
\text { For hospital length of } \\
\text { stay among infants born } \\
\text { premature at } 29-32 \text { wGA vs. } \\
33-35 \text { wGA and hospitalized } \\
\text { for RSV at }<12 \text { months }\end{array}$ \\
\hline $\begin{array}{l}\text { At-risk vs. } \\
\text { not-at-risk } \\
\text { population }\end{array}$ & $\begin{array}{l}\text { Prematurity: } \\
\text { 33-36 wGA }\end{array}$ & $\begin{array}{l}\text { Term: } \geq 37 \\
\text { wGA }\end{array}$ & $\begin{array}{l}\mathrm{RC}^{42}(\mathrm{n}=1) \\
7,597\end{array}$ & $\begin{array}{l}\text { MD } 1.00 \\
(0.88,1.12)\end{array}$ & N/A & $\begin{array}{l}\text { Moderate } \\
\text { to low } w^{b, c, d}\end{array}$ & $\begin{array}{l}\text { Small increase } \\
\text { For hospital length of } \\
\text { stay among infants born } \\
\text { premature at } 33-36 \text { wGA vs. } \\
\text { at term and hospitalized for } \\
\text { RSV at }<24 \text { months }\end{array}$ \\
\hline $\begin{array}{l}\text { At-risk vs. } \\
\text { not-at-risk } \\
\text { population }\end{array}$ & $\begin{array}{l}\text { Down } \\
\text { syndrome }\end{array}$ & $\begin{array}{l}\text { No Down } \\
\text { syndrome }\end{array}$ & $\begin{array}{l}\mathrm{RC}^{50}(\mathrm{n}=1) \\
7,206\end{array}$ & $\begin{array}{l}\text { MD } 3.00 \\
(1.95,4.05)\end{array}$ & N/A & Low $^{b, c}$ & $\begin{array}{l}\text { Small increase } \\
\text { For hospital length of stay } \\
\text { for RSV among infants with } \\
\text { vs. without Down syndrome } \\
\text { and hospitalized for RSV at } \\
<3 \text { years }\end{array}$ \\
\hline
\end{tabular}

Hospital length of stay, $<1$ day vs. $\geq 1$ day

\begin{tabular}{|c|c|c|c|c|c|c|c|c|}
\hline $\begin{array}{l}\text { At-risk } \\
\text { population }\end{array}$ & $\begin{array}{l}\text { Prematurity: } \\
29-32 \text { wGA }\end{array}$ & $\begin{array}{l}\text { Prematurity: } \\
\text { 33-36 wGA }\end{array}$ & $\begin{array}{l}\mathrm{RC}^{36}(\mathrm{n}=1) \\
542\end{array}$ & 13.9 per 100 & NS & $\begin{array}{l}<1 \text { day: } \\
\text { RR } 0.86 \\
(0.41 \\
1.78)\end{array}$ & $\operatorname{Low}^{c, e}$ & $\begin{array}{l}\text { Little to no difference } \\
\text { For hospital length of stay } \\
<1 \text { day among infants born } \\
\text { premature at } 29-32 \text { wGA vs. } \\
33-35 \text { wGA and hospitalized } \\
\text { in their first RSV season }\end{array}$ \\
\hline $\begin{array}{l}\text { At-risk } \\
\text { population }\end{array}$ & $\begin{array}{l}\text { Prematurity: } \\
\text { 29-32 wGA }\end{array}$ & $\begin{array}{l}\text { Prematurity: } \\
\text { 33-36 wGA }\end{array}$ & $\begin{array}{l}\mathrm{RC}^{36}(\mathrm{n}=1) ; \\
542\end{array}$ & 86.1 per 100 & NS & $\begin{array}{l}\geq 1 \text { day: } \\
\text { RR } 1.02 \\
(0.93 \\
1.13)\end{array}$ & Low $^{c, e}$ & $\begin{array}{l}\text { Little to no difference } \\
\text { For hospital length of stay } \\
\geq 1 \text { day among infants born } \\
\text { premature at } 29-32 \text { wGA vs. } \\
33-36 \text { wGA and hospitalized } \\
\text { in their first RSV season }\end{array}$ \\
\hline
\end{tabular}


Table 2: Summary of evidence for short-term outcomes among within-study population comparisons (continued)

\begin{tabular}{|c|c|c|c|c|c|c|c|c|}
\hline \multirow[b]{2}{*}{ Outcome } & \multirow[b]{2}{*}{$\begin{array}{c}\text { Comparator } \\
1\end{array}$} & \multirow[b]{2}{*}{$\begin{array}{c}\text { Comparator } \\
2\end{array}$} & \multirow{2}{*}{$\begin{array}{c}\text { Study } \\
\text { design } \\
\text { (no. of } \\
\text { studies); } \\
\text { Sample size }\end{array}$} & \multicolumn{2}{|c|}{$\begin{array}{c}\text { Absolute difference } \\
(95 \% \mathrm{CI})\end{array}$} & \multirow{2}{*}{$\begin{array}{c}\text { Relative } \\
\text { risk } \\
(95 \% \mathrm{Cl})\end{array}$} & \multirow{2}{*}{$\begin{array}{l}\text { Certainty } \\
\text { of } \\
\text { evidence }\end{array}$} & \multirow[b]{2}{*}{ Conclusion } \\
\hline & & & & $\begin{array}{c}\text { Comparator } \\
2 \text { risk }\end{array}$ & $\begin{array}{c}\text { Absolute } \\
\text { risk } \\
\text { difference }^{a}\end{array}$ & & & \\
\hline
\end{tabular}

ICU admission, among RSV-hospitalized population

\begin{tabular}{l|l|l|l|l|l|l|l|l} 
& & & & & & & $\begin{array}{l}\text { Little to no difference/very } \\
\text { uncertain }\end{array}$ \\
$\begin{array}{l}\text { At-risk } \\
\text { population }\end{array}$ & $\begin{array}{l}\text { Prematurity: } \\
29-32 \text { wGA }\end{array}$ & $\begin{array}{l}\text { Prematurity: } \\
33-35 \text { wGA }\end{array}$ & $\begin{array}{l}P^{26}(n=1) ; \\
212\end{array}$ & 50.4 per 100 & NS & $\begin{array}{l}\text { RR 1.03 } \\
(0.79, \\
1.34)\end{array}$ & $\begin{array}{l}\text { Low to } \\
\text { very } \\
\text { low } w^{b, c, d, e}\end{array}$ & $\begin{array}{l}\text { For ICU admission among } \\
\text { infants born premature at } \\
29-32 \text { wGA vs. 33-35 wGA } \\
\text { and hospitalized for RSV at } \\
<12 \text { months }\end{array}$ \\
\hline
\end{tabular}

ICU length of stay, mean days

\begin{tabular}{|c|c|c|c|c|c|c|c|}
\hline $\begin{array}{l}\text { At-risk } \\
\text { population }\end{array}$ & $\begin{array}{l}\text { Prematurity: } \\
\text { 29-32 wGA }\end{array}$ & $\begin{array}{l}\text { Prematurity: } \\
\text { 33-35 wGA }\end{array}$ & $\begin{array}{l}\mathrm{PC}^{26}(\mathrm{n}=1) \\
169\end{array}$ & $\begin{array}{l}\text { MD } 2.00 \\
(-0.28,4.28)\end{array}$ & N/A & $\begin{array}{l}\text { Low to } \\
\text { very } \\
\text { low }^{b, c, d, e}\end{array}$ & $\begin{array}{l}\text { Small increase/very uncertain } \\
\text { For ICU length of stay among } \\
\text { infants born premature at } \\
29-32 \text { wGA or at } 33-35 \text { wGA } \\
\text { and hospitalized for RSV at } \\
<12 \text { months }\end{array}$ \\
\hline
\end{tabular}

Mechanical ventilation, among RSV-hospitalized population

\begin{tabular}{|l|l|l|l|l|l|l|l|l|}
\hline $\begin{array}{l}\text { At-risk } \\
\text { population }\end{array}$ & $\begin{array}{l}\text { Prematurity: } \\
29-32 \text { wGA }\end{array}$ & $\begin{array}{l}\text { Prematurity: } \\
33-35 \text { wGA }\end{array}$ & $\begin{array}{l}\mathrm{PC}^{26}(\mathrm{n}=1) ; \\
212\end{array}$ & 17.1 per 100 & NS & $\begin{array}{l}\text { RR } 1.58 \\
(0.94, \\
2.65)\end{array}$ & $\begin{array}{l}\text { Lowall increase } \\
\text { For mechanical ventilation } \\
\text { among infants born } \\
\text { premature at 29-32 wGA vs. } \\
33-35 \text { wGA and hospitalized } \\
\text { for RSV at }<12 \text { months }\end{array}$ \\
\hline
\end{tabular}

Mechanical ventilation, among ICU population

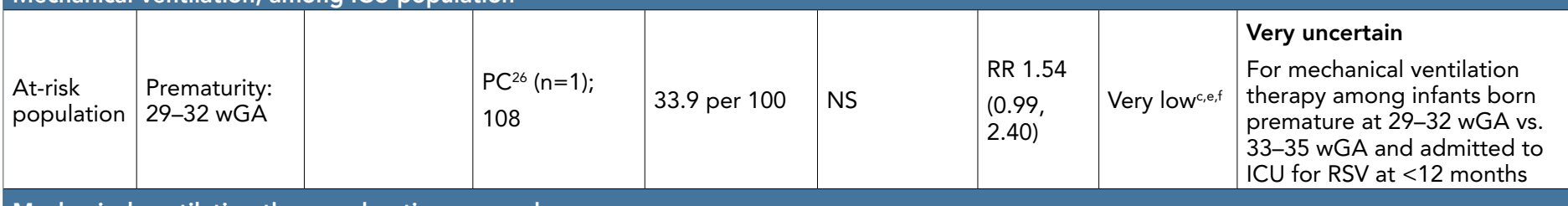

Mechanical ventilation therapy duration, mean days

\begin{tabular}{|c|c|c|c|c|c|c|c|}
\hline $\begin{array}{l}\text { At-risk } \\
\text { population }\end{array}$ & $\begin{array}{l}\text { Prematurity: } \\
\text { 29-32 wGA }\end{array}$ & $\begin{array}{l}\text { Prematurity: } \\
\text { 33-35 wGA }\end{array}$ & $\begin{array}{l}P^{26}(n=1) \\
45\end{array}$ & MD $2.00(-1.21,5.21)$ & N/A & Very low ${ }^{c, e, f}$ & $\begin{array}{l}\text { Very uncertain } \\
\text { For duration of mechanical } \\
\text { ventilation therapy among } \\
\text { infants born premature at } \\
29-32 \text { wGA vs. } 33-35 \text { wGA } \\
\text { and hospitalized for RSV at } \\
<12 \text { months }\end{array}$ \\
\hline
\end{tabular}

Case fatality, among RSV-hospitalized population

\begin{tabular}{|c|c|c|c|c|c|c|c|c|}
\hline $\begin{array}{l}\text { At-risk } \\
\text { population }\end{array}$ & $\begin{array}{l}\text { Prematurity: } \\
\text { 29-32 wGA }\end{array}$ & $\begin{array}{l}\text { Prematurity: } \\
\text { 33-35 wGA }\end{array}$ & $\begin{array}{l}P^{26}(n=1) ; \\
212\end{array}$ & 0 per 100 & NS & $\begin{array}{l}\text { RR 4.13 } \\
(0.17 \\
100.30)\end{array}$ & Very low ${ }^{c, e, f}$ & $\begin{array}{l}\text { Very uncertain } \\
\text { for death due to RSV among } \\
\text { infants born premature at } \\
29-32 \text { wGA vs. 33-35 wGA } \\
\text { and hospitalized for RSV at } \\
<12 \text { months }\end{array}$ \\
\hline \multicolumn{9}{|c|}{ Case fatality, among ICU population } \\
\hline $\begin{array}{l}\text { At-risk } \\
\text { population }\end{array}$ & $\begin{array}{l}\text { Prematurity: } \\
\text { 29-32 wGA }\end{array}$ & $\begin{array}{l}\text { Prematurity: } \\
\text { 33-35 wGA }\end{array}$ & $\begin{array}{l}P C^{26}(n=1) ; \\
108\end{array}$ & 0 per 100 & NS & $\begin{array}{l}\text { RR 4.02 } \\
(0.17 \\
96.53)\end{array}$ & Very low le,e,f $^{2}$ & $\begin{array}{l}\text { Very uncertain } \\
\text { For death due to RSV among } \\
\text { infants born premature at } \\
29-32 \text { wGA vs. } 33-35 \text { wGA } \\
\text { and admitted to ICU for RSV } \\
\text { at }<12 \text { months }\end{array}$ \\
\hline
\end{tabular}

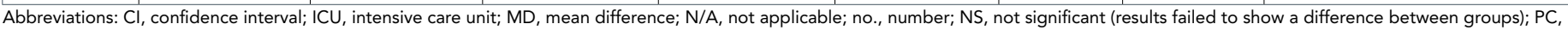
prospective cohort; RC, retrospective cohort; RFUPC, retrospective follow-up of prospective cohort; RR, relative risk; RSV, respiratory syncytial virus; vs., versus; wGA, weeks' gestational age

a Absolute risk reductions were calculated when findings were statistically significant; NS denotes when findings were not statistically significant

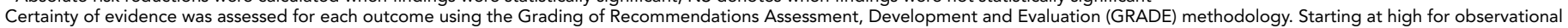

studies (for prognosis evidence) each outcome is rated as high, moderate, low or very low based on downgrading (if any) for one or more of the following domains:

b Study limitations, including selective outcome reporting

c Inconsistency

${ }^{d}$ Half decrement (-0.5) due to small concern for this domain

e Imprecision

${ }^{\mathrm{f}}$ Two decrements (-2) due to very serious concerns for this domain

Page $386 \quad$ CCDR • September $2021 \bullet$ Vol. 47 No. 9 
wGA $(25,30,32,36,42,43,48,51)$, respectively; $5.3 \%$ two years post-transplantation, $8.3 \%$ in the first two years of life, $12.3 \%$ in the first two years of life and $30.0 \%$ in the first or second RSV season for liver transplant (38), congenital cystic lung disease $(40)$, cystic fibrosis $(29,39)$ and childhood interstitial lung disease (35), respectively. The $95 \%$ confidence intervals for the latter three conditions were very wide. The pooled proportion for healthy term infants was $1.2 \%$ in the first two years of life $(37,42,45,52)$.

Case fatality rate attributable to RSV for those hospitalized were $1.1 \%(n=89), 2.5 \%(n=80), 4.4 \%(n=135)$ and $40.0 \%(n=10)$ for infants of 29-32 wGA (26), children residing in remote geographic area (46), children with liver transplant (38) and with leukemia (41), respectively. Most studies reported no attributable deaths. Many studies of clinical conditions contained very small sample sizes.

\section{Complications}

One study reported on complications associated with RSVhospitalization (Supplement 10).

\section{Long-term outcome comparisons from within-study comparisons}

Tables 3 to 5 summarize evidence for long-term outcomes from within-study comparisons. No study reported on growth or impaired development. Here we do not report on findings having very low certainty evidence.

\section{Table 3: Summary of evidence for wheeze associated with RSV infection among within-study population comparisons}

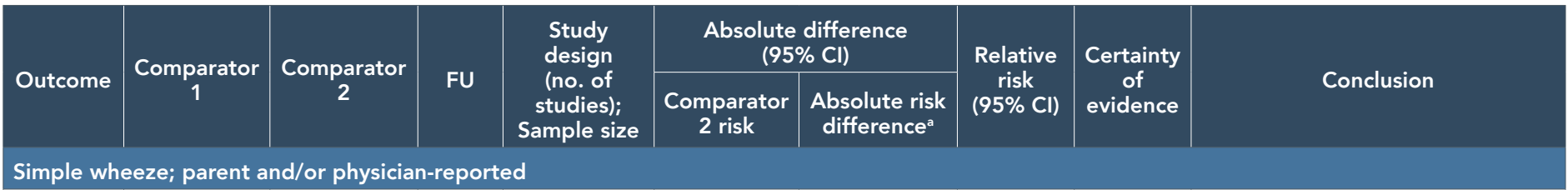

\begin{tabular}{|c|c|c|c|c|c|c|c|c|c|}
\hline $\begin{array}{l}\text { At-risk } \\
\text { with RSV-H } \\
\text { vs. at-risk } \\
\text { without } \\
\text { RSV-H }\end{array}$ & $\begin{array}{l}\text { Prematurity: } \\
32-35 \text { wGA, } \\
\text { RSV-H }<12 \\
\text { months of } \\
\text { age }\end{array}$ & $\begin{array}{l}\text { Prematurity: } \\
32-35 \text { wGA, } \\
\text { No RSV-H } \\
<12 \text { months } \\
\text { of age }\end{array}$ & $\begin{array}{l}\text { During } \\
6^{\text {th }} y\end{array}$ & $\begin{array}{l}P^{32}(n=1) \\
434\end{array}$ & 14 per 100 & NS & $\begin{array}{l}\text { RR } 1.16 \\
(0.70 \\
1.93)\end{array}$ & Low $^{b, c}$ & $\begin{array}{l}\text { Little to no difference } \\
\text { For parent/physician-reported simple } \\
\text { wheeze (episodes }<3 \text { within } 12 \text { months) } \\
\text { during the } 6^{\text {th }} \text { year among infants } \\
\text { born premature ( } 32-35 \text { wGA) with vs. } \\
\text { without hospitalization for RSV at }<12 \\
\text { months }\end{array}$ \\
\hline $\begin{array}{l}\text { At-risk } \\
\text { with RSV-H } \\
\text { vs. at-risk } \\
\text { without } \\
\text { RSV-H }\end{array}$ & $\begin{array}{l}\text { Prematurity: } \\
32-35 \text { wGA, } \\
\text { RSV-H }<12 \\
\text { months of } \\
\text { age }\end{array}$ & $\begin{array}{l}\text { Prematurity: } \\
32-35 \text { wGA, } \\
\text { No RSV-H } \\
<12 \text { months } \\
\text { of age }\end{array}$ & $\begin{array}{l}\text { Across } \\
2-6 \text { y }\end{array}$ & $\begin{array}{l}P^{32}(n=1) \\
474\end{array}$ & 49 per 100 & $\begin{array}{l}18 \text { more per } \\
100 \\
(7-30 \text { more })\end{array}$ & $\begin{array}{l}\text { RR 1.36 } \\
(1.15 \\
1.60)^{\prime}\end{array}$ & Low $^{b, c}$ & $\begin{array}{l}\text { Small increase } \\
\text { For parent/physician-reported simple } \\
\text { wheeze (episodes }<3 \text { within } 12 \text { months) } \\
\text { from } 2-6 \text { years among infants born } \\
\text { premature ( } 32-35 \text { wGA) with vs. } \\
\text { without hospitalization for RSV at }<12 \\
\text { months }\end{array}$ \\
\hline $\begin{array}{l}\text { At-risk } \\
\text { with RSV-H } \\
\text { vs. not-at- } \\
\text { risk with } \\
\text { RSV-H }\end{array}$ & $\begin{array}{l}\text { Prematurity: } \\
<33 \text { wGA \& } \\
\text { RSV-H }\end{array}$ & $\begin{array}{l}\text { Term: 39-41 } \\
\text { wGA \& RSV-H }\end{array}$ & $1 \mathrm{y}$ & $\begin{array}{l}\begin{array}{l}\text { RFUPC } 37 \\
(n=1) ; \\
17\end{array}\end{array}$ & 67 per 100 & NS & $\begin{array}{l}\text { RR 0.54 } \\
(0.18 \\
1.55)\end{array}$ & $\begin{array}{l}\text { Very } \\
\text { low }^{b, c, d}\end{array}$ & $\begin{array}{l}\text { Very uncertain } \\
\text { For parent and physician-reported } \\
\text { simple wheeze (episodes }<3 \text { in } 12 \\
\text { months) within one year among } \\
\text { premature }(<33 \text { wGA) vs. term infants } \\
\text { with hospitalization in their first RSV } \\
\text { season }\end{array}$ \\
\hline \multicolumn{10}{|c|}{ Recurrent wheeze; parent and/or physician-reported } \\
\hline $\begin{array}{l}\text { At-risk } \\
\text { with RSV-H } \\
\text { vs. at-risk } \\
\text { without } \\
\text { RSV-H }\end{array}$ & $\begin{array}{l}\text { Prematurity: } \\
32-35 \text { wGA, } \\
\text { RSV-H }<12 \\
\text { months of } \\
\text { age }\end{array}$ & $\begin{array}{l}\text { Prematurity: } \\
32-35 \text { wGA, } \\
\text { No RSV-H } \\
<12 \text { months } \\
\text { of age }\end{array}$ & $\begin{array}{l}\text { During } \\
6^{\text {th }} y\end{array}$ & $\begin{array}{l}P^{32}(n=1) \\
434\end{array}$ & 10 per 100 & NS & $\begin{array}{l}\text { RR } 1.28 \\
(0.71 \\
2.32)\end{array}$ & Low $^{b, c}$ & $\begin{array}{l}\text { Little to no difference } \\
\text { For parent/physician-reported } \\
\text { recurrent wheeze ( } \geq 3 \text { episodes within } \\
12 \text { months) during the } 6^{\text {th }} \text { year among } \\
\text { infants born premature ( } 32-35 \text { wGA) } \\
\text { with vs. without hospitalization for RSV } \\
\text { at }<12 \text { months }\end{array}$ \\
\hline $\begin{array}{l}\text { At-risk } \\
\text { with RSV-H } \\
\text { vs. at-risk } \\
\text { without } \\
\text { RSV-H }\end{array}$ & $\begin{array}{l}\text { Prematurity: } \\
32-35 \text { wGA, } \\
\text { RSV-H }<12 \\
\text { months of } \\
\text { age }\end{array}$ & $\begin{array}{l}\text { Prematurity: } \\
32-35 \text { wGA, } \\
\text { No RSV-H } \\
<12 \text { months } \\
\text { of age }\end{array}$ & $\begin{array}{l}\text { Across } \\
2-6 \text { y }\end{array}$ & $\begin{array}{l}P^{32}(n=1) \\
422\end{array}$ & 27 per 100 & $\begin{array}{l}19 \text { more per } \\
100 \\
(7-35 \text { more })\end{array}$ & $\begin{array}{l}\text { RR } 1.70 \\
(1.27 \\
2.29)\end{array}$ & Low $^{b, c}$ & $\begin{array}{l}\text { Small increase } \\
\text { For parent/physician-reported } \\
\text { recurrent wheeze ( } \geq 3 \text { episodes within } \\
12 \text { months) from } 2-6 \text { years among } \\
\text { infants born premature ( } 32-35 \text { wGA) } \\
\text { with vs. without hospitalization for RSV } \\
\text { at }<12 \text { months }\end{array}$ \\
\hline $\begin{array}{l}\text { At-risk with } \\
\text { RSV-H vs. } \\
\text { not-at-risk } \\
\text { with RSV-H }\end{array}$ & $\begin{array}{l}\text { Prematurity: } \\
<33 \text { wGA \& } \\
\text { RSV-H }\end{array}$ & $\begin{array}{l}\text { Term: } 39-41 \\
\text { wGA \& RSV-H }\end{array}$ & $1 \mathrm{y}$ & $\begin{array}{l}\text { RFUPC } \\
(n=1) ; \\
17\end{array}$ & 0 per 100 & NS & $\begin{array}{l}\text { RR } 0.80 \\
(0.04 \\
16.14)\end{array}$ & $\begin{array}{l}\text { Very } \\
\text { low }^{b, c, d}\end{array}$ & $\begin{array}{l}\text { Very uncertain } \\
\text { For parent and physician-reported } \\
\text { recurrent wheeze } \geq 3 \text { episodes in } \\
12 \text { months) within one year among } \\
\text { premature }(<33 \text { wGA) vs. term infants } \\
\text { with hospitalization in their first RSV } \\
\text { season }\end{array}$ \\
\hline
\end{tabular}


Table 3: Summary of evidence for wheeze associated with RSV infection among within-study population comparisons (continued)

\begin{tabular}{|c|c|c|c|c|c|c|c|c|c|}
\hline \multirow{2}{*}{ Outcome } & \multirow{2}{*}{$\begin{array}{c}\text { Comparator } \\
1\end{array}$} & \multirow{2}{*}{$\begin{array}{c}\text { Comparator } \\
2\end{array}$} & \multirow{2}{*}{ FU } & \multirow{2}{*}{$\begin{array}{c}\text { Study } \\
\text { design } \\
\text { (no. of } \\
\text { studies); } \\
\text { Sample size }\end{array}$} & \multicolumn{2}{|c|}{$\begin{array}{l}\text { Absolute difference } \\
(95 \% \mathrm{CI})\end{array}$} & \multirow{2}{*}{$\begin{array}{c}\text { Relative } \\
\text { risk } \\
(95 \% \mathrm{Cl})\end{array}$} & \multirow{2}{*}{$\begin{array}{c}\text { Certainty } \\
\text { of } \\
\text { evidence }\end{array}$} & \multirow{2}{*}{ Conclusion } \\
\hline & & & & & $\begin{array}{c}\text { Comparator } \\
2 \text { risk }\end{array}$ & $\begin{array}{l}\text { Absolute risk } \\
\text { difference }^{\mathrm{a}}\end{array}$ & & & \\
\hline
\end{tabular}

\begin{tabular}{|c|c|c|c|c|c|c|c|c|c|}
\hline $\begin{array}{l}\text { At-risk } \\
\text { with RSV-H } \\
\text { vs. at-risk } \\
\text { without } \\
\text { RSV-H }\end{array}$ & $\begin{array}{l}\text { Prematurity: } \\
32-35 \text { wGA, } \\
\text { RSV-H }<12 \\
\text { months of } \\
\text { age }\end{array}$ & $\begin{array}{l}\text { Prematurity: } \\
\text { 32-35 wGA, } \\
\text { No RSV-H } \\
<12 \text { months } \\
\text { of age }\end{array}$ & $\begin{array}{l}\text { During } \\
6^{\text {th }} y\end{array}$ & $\begin{array}{l}P C^{34}(n=1) ; \\
434\end{array}$ & 9 per 100 & NS & $\begin{array}{l}\text { RR } 0.91 \\
(0.44 \\
1.88)\end{array}$ & Low $^{b, c}$ & $\begin{array}{l}\text { Little to no difference } \\
\text { For parent/physician-reported severe } \\
\text { wheeze ( } \geq 1 \text { hospitalization or } \geq 3 \\
\text { medical attendances or medication } \\
\text { for three consecutive months or five } \\
\text { cumulative months) during the } 6^{\text {th }} \text { year } \\
\text { among infants born premature ( } 32-35 \\
\text { wGA) with vs. without hospitalization } \\
\text { for RSV at }<12 \text { months }\end{array}$ \\
\hline $\begin{array}{l}\text { At-risk } \\
\text { with RSV-H } \\
\text { vs. at-risk } \\
\text { without } \\
\text { RSV-H }\end{array}$ & $\begin{array}{l}\text { Prematurity: } \\
32-35 \text { wGA, } \\
\text { RSV-H }<12 \\
\text { months of } \\
\text { age }\end{array}$ & $\begin{array}{l}\text { Prematurity: } \\
\text { 32-35 wGA, } \\
\text { No RSV-H } \\
<12 \text { months } \\
\text { of age }\end{array}$ & $\begin{array}{l}\text { Across } \\
2-6 y\end{array}$ & $\begin{array}{l}P C^{34}(n=1) ; \\
427\end{array}$ & 24 per 100 & $\begin{array}{l}14 \text { more } \\
\text { per } 100 \\
\text { (3-29 more) }\end{array}$ & $\begin{array}{l}\text { RR 1.59 } \\
(1.13, \\
2.24)\end{array}$ & Low $^{b, c}$ & $\begin{array}{l}\text { Small increase } \\
\text { For parent/physician-reported severe } \\
\text { wheeze ( } \geq 1 \text { hospitalization or } \geq 3 \\
\text { medical attendances or medication } \\
\text { for three consecutive months or five } \\
\text { cumulative months) from } 2-6 \text { years } \\
\text { among infants born premature ( } 32-35 \\
\text { wGA) with vs. without hospitalization } \\
\text { for RSV at }<12 \text { months }\end{array}$ \\
\hline $\begin{array}{l}\text { At-risk } \\
\text { with RSV-H } \\
\text { vs. not-at- } \\
\text { risk with } \\
\text { RSV-H }\end{array}$ & $\begin{array}{l}\text { Prematurity: } \\
<33 \text { wGA \& } \\
\text { RSV-H }\end{array}$ & $\begin{array}{l}\text { Term: 39-41 } \\
\text { wGA \& RSV-H }\end{array}$ & $1 \mathrm{y}$ & $\begin{array}{l}\text { RFUPC } 37 \\
(n=1) ; \\
17\end{array}$ & 0 per 100 & NS & $\begin{array}{l}\text { RD } 0.00 \\
(-0.34 \\
0.34)\end{array}$ & $\begin{array}{l}\text { Very } \\
\text { low }^{\mathrm{b}, c, \mathrm{~d}}\end{array}$ & $\begin{array}{l}\text { Very uncertain } \\
\text { For physician-reported severe } \\
\text { wheeze (hospitalization for wheeze } \\
\text { in } 12 \text { months) within one year among } \\
\text { premature (<33 wGA) vs. term infants } \\
\text { with hospitalization in their first RSV } \\
\text { season }\end{array}$ \\
\hline \multicolumn{10}{|c|}{ Wheeze duration (days per month post-RSV); parent-reported } \\
\hline $\begin{array}{l}\text { Not-at-risk } \\
\text { population }\end{array}$ & $\begin{array}{l}\text { RSV-positive, } \\
\text { hospitalized }\end{array}$ & $\begin{array}{l}\text { RSV-positive, } \\
\text { non- } \\
\text { hospitalized }\end{array}$ & $1 \mathrm{y}$ & $\begin{array}{l}P C^{52}(n=1) \\
90\end{array}$ & $\begin{array}{l}\text { MD } 0.70 \\
(-0.94,2.34)\end{array}$ & & N/A & $\begin{array}{l}\text { Very } \\
\text { low }^{b, c, d}\end{array}$ & $\begin{array}{l}\text { Very uncertain } \\
\text { For parent-reported days with wheeze } \\
\text { at one year among hospitalized vs. } \\
\text { non-hospitalized healthy term infants } \\
\text { positive for RSV at }<12 \text { months }\end{array}$ \\
\hline
\end{tabular}

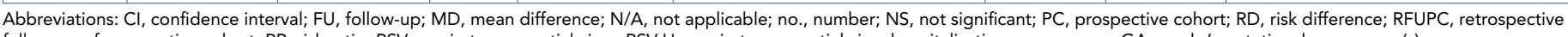
follow-up of prospective cohort; RR, risk ratio; RSV, respiratory syncytial virus; RSV-H, respiratory syncytial virus hospitalization; vs., versus; wGA: weeks' gestational age; y, year(s)

a Absolute risk reductions were calculated when findings were statistically significant; NS denotes when findings were not statistically significant

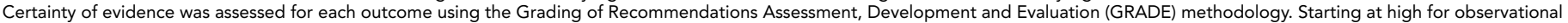
studies (for prognosis evidence) each outcome is rated as high, moderate, low or very low based on downgrading (if any) for one or more of the following domains:

b Inconsistency

c Imprecision

d Two decrements (-2) due to very serious concerns for this domain 
Table 4: Summary of evidence for asthma associated with RSV infection among within-study population comparisons

\begin{tabular}{|c|c|c|c|c|c|c|c|c|c|}
\hline \multirow{2}{*}{ Outcome } & \multirow{2}{*}{$\begin{array}{c}\text { Comparator } \\
1\end{array}$} & \multirow{2}{*}{$\begin{array}{c}\text { Comparator } \\
2\end{array}$} & \multirow{2}{*}{ FU } & \multirow{2}{*}{$\begin{array}{l}\text { Study design } \\
\text { (no. of } \\
\text { studies); } \\
\text { Sample size }\end{array}$} & \multicolumn{2}{|c|}{$\begin{array}{l}\text { Absolute difference } \\
(95 \% \mathrm{Cl})\end{array}$} & \multirow{2}{*}{$\begin{array}{l}\text { Relative risk } \\
(95 \% \mathrm{Cl})\end{array}$} & \multirow{2}{*}{$\begin{array}{c}\text { Certainty } \\
\text { of } \\
\text { evidence }\end{array}$} & \multirow{2}{*}{ Conclusion } \\
\hline & & & & & $\begin{array}{c}\text { Comparator } \\
2 \text { risk }\end{array}$ & $\begin{array}{l}\text { Absolute risk } \\
\text { difference }^{a}\end{array}$ & & & \\
\hline \multicolumn{10}{|c|}{ Asthma; physician-diagnosed } \\
\hline $\begin{array}{l}\text { Not-at-risk } \\
\text { population }\end{array}$ & $\begin{array}{l}\text { RSV infection } \\
\text { in first year } \\
\text { of life }\end{array}$ & $\begin{array}{l}\text { Infection with } \\
\text { a respiratory } \\
\text { pathogen } \\
\text { other than } \\
\text { RSV in first } \\
\text { year of life }\end{array}$ & $7 y$ & $\begin{array}{l}P^{31}(n=1) \\
329\end{array}$ & 12 per 100 & $\begin{array}{l}15 \text { more per } 100 \\
(4-35 \text { more) }\end{array}$ & $\begin{array}{l}\text { RR } 2.33 \\
(1.35,4.05) \\
\text { Adjusted for } \\
\text { total number } \\
\text { of respiratory } \\
\text { episodes: } \\
\text { OR } 1.26 \\
(0.54,2.91) \\
p=0.59\end{array}$ & $\begin{array}{l}\text { Very } \\
\text { low }^{b, c, d, e}\end{array}$ & $\begin{array}{l}\text { Very uncertain } \\
\text { For physician-diagnosed } \\
\text { asthma at seven years of } \\
\text { age among healthy infants } \\
\text { with RSV vs. a different } \\
\text { respiratory pathogen in } \\
\text { the first year of life }\end{array}$ \\
\hline $\begin{array}{l}\text { Not-at-risk } \\
\text { population }\end{array}$ & RSV-H & No RSV-H & $28-31$ y & $\begin{array}{l}\mathrm{PC}^{28}(\mathrm{n}=1) \\
129\end{array}$ & 13 per 100 & NS & $\begin{array}{l}\text { RR } 1.82 \\
(0.84,3.94)\end{array}$ & Very low $w^{b, c, e}$ & $\begin{array}{l}\text { Very uncertain } \\
\text { For physician-diagnosed } \\
\text { asthma at } 28-31 \text { years } \\
\text { of age among term } \\
\text { infants with vs. without } \\
\text { hospitalization for RSV at } \\
\text { age }<24 \text { months }\end{array}$ \\
\hline \multicolumn{10}{|c|}{ Asthma; self-reported } \\
\hline $\begin{array}{l}\text { Not-at-risk } \\
\text { population }\end{array}$ & RSV-H & No RSV-H & $\begin{array}{l}17-20 y \\
28-31 y\end{array}$ & $\begin{array}{l}P^{27,28}(n=2) \\
203\end{array}$ & 15 per 100 & $\begin{array}{l}19 \text { more per } 100 \\
(0.1-60 \text { more })\end{array}$ & $\begin{array}{l}\text { RR } 2.28 \\
(1.01,5.12)\end{array}$ & Low $^{b, e}$ & $\begin{array}{l}\text { Small increase } \\
\text { For self-reported asthma } \\
\text { in adulthood ( } 17-31 \text { years } \\
\text { of age) among infants with } \\
\text { vs. without hospitalization } \\
\text { for RSV at age }<24 \text { months }\end{array}$ \\
\hline
\end{tabular}

Asthma medication (bronchodilator)

\begin{tabular}{|c|c|c|c|c|c|c|c|c|c|}
\hline $\begin{array}{l}\text { At-risk } \\
\text { with RSV-H } \\
\text { vs. at-risk } \\
\text { without } \\
\text { RSV-H }\end{array}$ & $\begin{array}{l}\text { Prematurity: } \\
32-35 \text { wGA, } \\
\text { RSV-H }\end{array}$ & $\begin{array}{l}\text { Prematurity: } \\
32-35 \text { wGA, } \\
\text { No RSV-H }\end{array}$ & $\begin{array}{l}\text { Across } \\
2-6 y\end{array}$ & $\begin{array}{l}\operatorname{PC}^{32}(n=1) ; \\
487\end{array}$ & 17 per 100 & $\begin{array}{l}8 \text { more per } 100 \\
(4-13 \text { more })\end{array}$ & $\begin{array}{l}\text { RR } 1.48 \\
(1.23,1.77)\end{array}$ & Low $^{c, e}$ & $\begin{array}{l}\text { Small increase } \\
\text { Parent-reported } \\
\text { bronchodilator use from } \\
2-6 \text { years of age among } \\
\text { infants born premature } \\
\text { ( } 32-35 \text { wGA) with vs. } \\
\text { without hospitalization for } \\
\text { RSV at }<12 \text { months }\end{array}$ \\
\hline $\begin{array}{l}\text { Not-at-risk } \\
\text { population }\end{array}$ & RSV-H & No RSV-H & $28-31$ y & $\begin{array}{l}P^{28}(n=1) ; \\
129\end{array}$ & 14 per 100 & $\begin{array}{l}16 \text { more per } 100 \\
(1-47 \text { more })\end{array}$ & $\begin{array}{l}\text { RR } 2.17 \\
(1.08,4.34)\end{array}$ & Very low bic,e $^{\text {b }}$ & $\begin{array}{l}\text { Very uncertain } \\
\text { For self-reported } \\
\text { bronchodilator use in } \\
\text { adulthood ( } 28-31 \text { years } \\
\text { of age) among term } \\
\text { infants with vs. without } \\
\text { hospitalization for RSV at } \\
\text { age }<24 \text { months }\end{array}$ \\
\hline \multicolumn{10}{|c|}{ Asthma medication (inhaled CS) } \\
\hline $\begin{array}{l}\text { At-risk } \\
\text { with RSV-H } \\
\text { vs. at-risk } \\
\text { without } \\
\text { RSV-H }\end{array}$ & $\begin{array}{l}\text { Prematurity: } \\
32-35 \text { wGA, } \\
\text { RSV-H }\end{array}$ & $\begin{array}{l}\text { Prematurity: } \\
\text { 32-35 wGA, } \\
\text { No RSV-H }\end{array}$ & $\begin{array}{l}\text { Across } \\
2-6 y\end{array}$ & $\begin{array}{l}P^{32}(n=1) ; \\
487\end{array}$ & 16 per 100 & $\begin{array}{l}10 \text { more per } 100 \\
(2-22 \text { more })\end{array}$ & $\begin{array}{l}\text { RR } 1.65 \\
(1.13,2.40)\end{array}$ & Low $^{c, e}$ & $\begin{array}{l}\text { Small increase } \\
\text { Parent-reported ICS } \\
\text { use from } 2-6 \text { years of } \\
\text { age among infants born } \\
\text { premature ( } 32-35 \text { wGA) } \\
\text { with hospitalization for } \\
\text { RSV at }<12 \text { months }\end{array}$ \\
\hline $\begin{array}{l}\text { Not-at-risk } \\
\text { population }\end{array}$ & RSV-H & No RSV-H & $28-31$ y & $\begin{array}{l}P^{28}(n=1) ; \\
129\end{array}$ & 11 per 100 & NS & $\begin{array}{l}\text { RR } 1.56 \\
(0.62,3.89)\end{array}$ & Very low ${ }^{b, c, e}$ & $\begin{array}{l}\text { Very uncertain } \\
\text { For self-reported ICS use } \\
\text { in adulthood ( } 28-31 \text { years } \\
\text { of age) among term } \\
\text { infants with vs. without } \\
\text { hospitalization for RSV at } \\
\text { age }<24 \text { months }\end{array}$ \\
\hline
\end{tabular}


Table 4: Summary of evidence for asthma associated with RSV infection among within-study population comparisons (continued)

\begin{tabular}{|c|c|c|c|c|c|c|c|c|c|}
\hline \multirow{2}{*}{ Outcome } & \multirow{2}{*}{$\begin{array}{c}\text { Comparator } \\
1\end{array}$} & \multirow{2}{*}{$\begin{array}{c}\text { Comparator } \\
2\end{array}$} & \multirow{2}{*}{ FU } & \multirow{2}{*}{$\begin{array}{l}\text { Study design } \\
\text { (no. of } \\
\text { studies); } \\
\text { Sample size }\end{array}$} & \multicolumn{2}{|c|}{$\begin{array}{l}\text { Absolute difference } \\
(95 \% \mathrm{Cl})\end{array}$} & \multirow{2}{*}{$\begin{array}{l}\text { Relative risk } \\
(95 \% \mathrm{Cl})\end{array}$} & \multirow{2}{*}{$\begin{array}{l}\text { Certainty } \\
\text { of } \\
\text { evidence }\end{array}$} & \multirow{2}{*}{ Conclusion } \\
\hline & & & & & $\begin{array}{l}\text { Comparator } \\
2 \text { risk }\end{array}$ & $\begin{array}{l}\text { Absolute risk } \\
\text { difference }^{a}\end{array}$ & & & \\
\hline \multicolumn{10}{|c|}{ Asthma medication (leukotriene antagonist) } \\
\hline $\begin{array}{l}\text { At-risk } \\
\text { with RSV-H } \\
\text { vs. at-risk } \\
\text { without } \\
\text { RSV-H }\end{array}$ & $\begin{array}{l}\text { Prematurity: } \\
\text { 32-35 wGA, } \\
\text { RSV-H }\end{array}$ & $\begin{array}{l}\text { Prematurity: } \\
32-35 \text { wGA, } \\
\text { No RSV-H }\end{array}$ & $\begin{array}{l}\text { Across } \\
2-6 y\end{array}$ & $\begin{array}{l}P^{32}(n=1) \\
487\end{array}$ & 6 per 100 & $\begin{array}{l}10 \text { more per } 100 \\
(3-22 \text { more) }\end{array}$ & $\begin{array}{l}\text { RR } 2.52 \\
(1.43,4.42)\end{array}$ & Low $^{c, e}$ & $\begin{array}{l}\text { Increased } \\
\text { Parent-reported } \\
\text { leukotriene antagonist } \\
\text { use from 2-6 years of } \\
\text { age among infants born } \\
\text { premature ( } 32-35 \text { wGA) } \\
\text { with vs. without } \\
\text { hospitalization for RSV at } \\
<12 \text { months }\end{array}$ \\
\hline
\end{tabular}

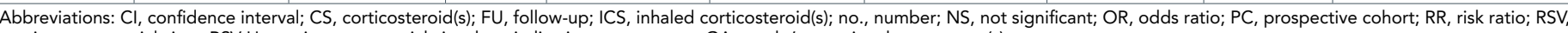
respiratory syncytial virus; RSV-H, respiratory syncytial virus hospitalization; vs.: versus; wGA, weeks' gestational age; $y$, year(s)

a Absolute risk reductions were calculated when findings were statistically significant; NS denotes when findings were not statistically significant

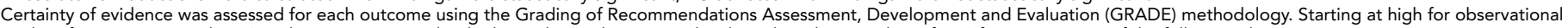
studies (for prognosis evidence) each outcome is rated as high, moderate, low or very low based on downgrading (if any) for one or more of the following domains:

b Study limitations, including selective outcome reporting

c Inconsistency

Indirectness

Imprecision

Half decrement $(-0.5)$ due to small concern for this domain

Table 5: Summary of evidence for lung function associated with RSV infection among within-study population comparisons

\begin{tabular}{|c|c|c|c|c|c|c|c|c|}
\hline \multirow[b]{2}{*}{ Outcome } & \multirow[b]{2}{*}{$\begin{array}{c}\text { Comparator } \\
1\end{array}$} & \multirow[b]{2}{*}{$\begin{array}{c}\text { Comparator } \\
2\end{array}$} & \multirow[b]{2}{*}{ FU } & \multirow{2}{*}{$\begin{array}{c}\text { Study design } \\
\text { (no. of } \\
\text { studies); } \\
\text { Sample size }\end{array}$} & \multicolumn{2}{|c|}{$\begin{array}{l}\text { Absolute difference } \\
(95 \% \mathrm{CI})\end{array}$} & \multirow{2}{*}{$\begin{array}{c}\text { Relative } \\
\text { risk } \\
(95 \% \mathrm{Cl})\end{array}$} & \multirow{2}{*}{$\begin{array}{l}\text { Certainty } \\
\text { of } \\
\text { evidence }\end{array}$} \\
\hline & & & & & $\begin{array}{l}\text { Comparator } \\
2 \text { risk }\end{array}$ & $\begin{array}{l}\text { Absolute } \\
\text { risk } \\
\text { difference }^{a}\end{array}$ & & \\
\hline
\end{tabular}

Lung function: FEV 1 Z-score ranking $[-2,-1]$

\begin{tabular}{|c|c|c|c|c|c|c|c|c|c|}
\hline $\begin{array}{l}\text { At-risk } \\
\text { with RSV-H } \\
\text { vs. at-risk } \\
\text { without } \\
\text { RSV-H }\end{array}$ & $\begin{array}{l}\text { Prematurity: } \\
32-35 \text { wGA, } \\
\text { RSV-H }\end{array}$ & $\begin{array}{l}\text { Prematurity: } \\
32-35 \text { wGA, } \\
\text { No RSV-H }\end{array}$ & $\begin{array}{l}\text { During } \\
6^{\text {th }} y\end{array}$ & $\begin{array}{l}P^{32}(n=1) ; \\
243\end{array}$ & 21 per 100 & NS & $\begin{array}{l}\text { RR } 0.83 \\
(0.45,1.53)\end{array}$ & Low $^{b, c}$ & $\begin{array}{l}\text { Little to no difference } \\
\text { For forced expiratory volume in } \\
\text { one second (Z-score rank of [-2, } \\
-1] \text {, considered extreme range) } \\
\text { during the } 6^{\text {th }} \text { year of age among } \\
\text { children hospitalized with RSV at } \\
<12 \text { months }\end{array}$ \\
\hline
\end{tabular}

Lung function (FEV 1 pre-BD, mean $\%$ of predicted)

\begin{tabular}{|c|c|c|c|c|c|c|c|c|}
\hline $\begin{array}{l}\text { Not-at-risk } \\
\text { population }\end{array}$ & RSV-H & No RSV-H & $\begin{array}{l}17- \\
20 y ; \\
28-31 \text { y }\end{array}$ & $\begin{array}{l}P^{27,28}(n=2) ; \\
202\end{array}$ & $\begin{array}{l}\text { MD -7.63 } \\
(-11.35,-3.91)\end{array}$ & N/A & $\operatorname{Low}^{c, d}$ & $\begin{array}{l}\text { Small decrease } \\
\text { For forced expiratory volume } \\
\text { in one second (mean \% of } \\
\text { predicted, pre-bronchodilation } \\
\text { test) in adulthood ( } 17-31 \text { years } \\
\text { of age) among infants with vs. } \\
\text { without hospitalization for RSV at } \\
\text { age }<24 \text { months }\end{array}$ \\
\hline
\end{tabular}

Lung function (FEV ${ }_{1}$, change in mean $\%$ predicted)

\begin{tabular}{|c|c|c|c|c|c|c|c|c|}
\hline $\begin{array}{l}\text { Not-at-risk } \\
\text { population }\end{array}$ & RSV-H & No RSV-H & $\begin{array}{l}17- \\
20 y ; \\
28-31 \text { y }\end{array}$ & $\begin{array}{l}P^{27,28}(n=2) ; \\
202\end{array}$ & $\begin{array}{l}\text { MD } 0.81 \\
(-0.67,2.30)\end{array}$ & $\mathrm{N} / \mathrm{A}$ & $\operatorname{Low}^{c, d}$ & $\begin{array}{l}\text { Little to no difference } \\
\text { For forced expiratory volume } \\
\text { in one second (change in } \\
\text { mean \% predicted, pre vs. } \\
\text { post-bronchodilation test) in } \\
\text { adulthood (17-31 years of age) } \\
\text { among infants with vs. without } \\
\text { hospitalization for RSV at age } \\
<24 \text { months }\end{array}$ \\
\hline
\end{tabular}

Lung function (FVC pre-BD, mean \% of predicted)

\begin{tabular}{|c|c|c|c|c|c|c|c|c|}
\hline $\begin{array}{l}\text { Not-at-risk } \\
\text { population }\end{array}$ & RSV-H & No RSV-H & $\begin{array}{l}17- \\
20 y ; \\
28-31 \text { y }\end{array}$ & $\begin{array}{l}P^{27,28}(n=2) \\
202\end{array}$ & $\begin{array}{l}\text { MD }-4.74 \\
(-7.80,-1.67)\end{array}$ & N/A & $\operatorname{Low}^{c, d}$ & $\begin{array}{l}\text { Small decrease } \\
\text { For forced vital capacity } \\
\text { (mean \% of predicted, pre- } \\
\text { bronchodilation test) in } \\
\text { adulthood (17-31 years of age) } \\
\text { among infants with vs. without } \\
\text { hospitalization for RSV at age } \\
<24 \text { months }\end{array}$ \\
\hline
\end{tabular}


Table 5: Summary of evidence for lung function associated with RSV infection among within-study population comparisons (continued)

\begin{tabular}{|c|c|c|c|c|c|c|c|c|c|}
\hline \multirow[b]{2}{*}{ Outcome } & \multirow[b]{2}{*}{$\begin{array}{c}\text { Comparator } \\
1\end{array}$} & \multirow[b]{2}{*}{$\begin{array}{c}\text { Comparator } \\
2\end{array}$} & \multirow[b]{2}{*}{ FU } & \multirow{2}{*}{$\begin{array}{l}\text { Study design } \\
\text { (no. of } \\
\text { studies); } \\
\text { Sample size }\end{array}$} & \multicolumn{2}{|c|}{$\begin{array}{c}\text { Absolute difference } \\
(95 \% \mathrm{Cl})\end{array}$} & \multirow{2}{*}{$\begin{array}{c}\text { Relative } \\
\text { risk } \\
(95 \% \mathrm{Cl})\end{array}$} & \multirow{2}{*}{$\begin{array}{l}\text { Certainty } \\
\text { of } \\
\text { evidence }\end{array}$} & \multirow[b]{2}{*}{ Conclusion } \\
\hline & & & & & $\begin{array}{l}\text { Comparator } \\
2 \text { risk }\end{array}$ & $\begin{array}{l}\text { Absolute } \\
\text { risk } \\
\text { difference }^{a}\end{array}$ & & & \\
\hline
\end{tabular}

\begin{tabular}{|c|c|c|c|c|c|c|c|c|}
\hline $\begin{array}{l}\text { Not-at-risk } \\
\text { population }\end{array}$ & RSV-H & No RSV-H & $17-20 y$ & $\begin{array}{l}P^{27}(n=1) \\
74\end{array}$ & $\begin{array}{l}\text { MD } 0.60 \\
(-0.67,1.87)\end{array}$ & N/A & $\begin{array}{l}\text { Very } \\
\text { low }{ }^{c, d, e}\end{array}$ & $\begin{array}{l}\text { Very uncertain } \\
\text { For forced vital capacity (change } \\
\text { in mean \% predicted, pre vs. } \\
\text { post-bronchodilation test) in } \\
\text { adulthood ( } 17-20 \text { years of age) } \\
\text { among infants with vs. without } \\
\text { hospitalization for RSV at age } \\
<24 \text { months }\end{array}$ \\
\hline $\begin{array}{l}\text { Not-at-risk } \\
\text { population }\end{array}$ & RSV-H & No RSV-H & $\begin{array}{l}17- \\
20 y ; \\
28-31 \text { y }\end{array}$ & $\begin{array}{l}P^{27,28}(n=2) ; \\
202\end{array}$ & $\begin{array}{l}\text { MD }-3.20 \\
(-9.07,2.67)\end{array}$ & $\mathrm{N} / \mathrm{A}$ & $\begin{array}{l}\text { Very } \\
\text { low }{ }^{b, c, d}\end{array}$ & $\begin{array}{l}\text { Very uncertain } \\
\text { For } \mathrm{FEV}_{1} / \mathrm{FVC} \text { (mean } \% \text { of } \\
\text { predicted, pre-bronchodilation } \\
\text { test) in adulthood ( } 17-31 \text { years } \\
\text { of age) among infants with vs. } \\
\text { without hospitalization for RSV at } \\
\text { age }<24 \text { months }\end{array}$ \\
\hline
\end{tabular}

Lung function ( $\mathrm{FEV}_{1} / \mathrm{FVC}$, change in mean $\%$ predicted)

\begin{tabular}{|c|c|c|c|c|c|c|c|c|}
\hline $\begin{array}{l}\text { Not-at-risk } \\
\text { population }\end{array}$ & RSV-H & No RSV-H & $17-20 y$ & $\begin{array}{l}\mathrm{PC}^{27}(\mathrm{n}=1) \\
74\end{array}$ & $\begin{array}{l}\text { MD }-0.20 \\
(-2.71,2.31)\end{array}$ & N/A & $\begin{array}{l}\text { Very } \\
\text { low }^{b, c, e}\end{array}$ & $\begin{array}{l}\text { Very uncertain } \\
\text { For } \mathrm{FEV}_{1} / \mathrm{FVC} \text { (change in } \\
\text { mean } \% \text { predicted, pre vs. } \\
\text { post-bronchodilation test) in } \\
\text { adulthood ( } 17-20 \text { years of age) } \\
\text { among infants with vs. without } \\
\text { hospitalization for RSV at age } \\
<24 \text { months }\end{array}$ \\
\hline
\end{tabular}

Lung function (FENO, mean ppb)

\begin{tabular}{|c|c|c|c|c|c|c|c|c|}
\hline $\begin{array}{l}\text { Not-at-risk } \\
\text { population }\end{array}$ & RSV-H & No RSV-H & $\begin{array}{l}17- \\
20 y ; \\
28-31 \text { y }\end{array}$ & $\begin{array}{l}P^{27,28}(n=2) ; \\
202\end{array}$ & $\begin{array}{l}\text { MD -1.00 } \\
(-14.49,12.49)\end{array}$ & N/A & Low $^{c, d}$ & $\begin{array}{l}\text { Little to no difference } \\
\text { For fractional exhaled nitric } \\
\text { oxide (mean ppb) in adulthood } \\
\text { (17-31 years of age) among } \\
\text { infants with vs. without } \\
\text { hospitalization for RSV at age } \\
<24 \text { months }\end{array}$ \\
\hline
\end{tabular}

Lung function (MEF50 pre-BD, mean $\%$ of predicted)

\begin{tabular}{|c|c|c|c|c|c|c|c|c|}
\hline $\begin{array}{l}\text { Not-at-risk } \\
\text { population }\end{array}$ & RSV-H & No RSV-H & $17-20 y$ & $\begin{array}{l}P^{27}(n=1) ; \\
74\end{array}$ & $\begin{array}{l}\text { MD -4.00 } \\
(-14.95,6.95)\end{array}$ & N/A & $\begin{array}{l}\text { Very } \\
\operatorname{low}^{b, c, d, e}\end{array}$ & $\begin{array}{l}\text { Very uncertain } \\
\text { For maximum expiratory flow } \\
\text { after } 50 \% \text { of expired FVC } \\
\text { (change in mean \% predicted, } \\
\text { pre-bronchodilation test) in } \\
\text { adulthood (17-20 years of age) } \\
\text { among infants with vs. without } \\
\text { hospitalization for RSV at age } \\
<24 \text { months }\end{array}$ \\
\hline
\end{tabular}

Lung function (MEF50, change in mean \% predicted)

\begin{tabular}{|c|c|c|c|c|c|c|c|c|}
\hline $\begin{array}{l}\text { Not-at-risk } \\
\text { population }\end{array}$ & RSV-H & No RSV-H & $17-20 y$ & $\begin{array}{l}P^{27}(n=1) ; \\
74\end{array}$ & $\begin{array}{l}\text { MD } 3.70 \\
(-5.42,12.82)\end{array}$ & $\mathrm{N} / \mathrm{A}$ & $\begin{array}{l}\text { Very } \\
\text { low }^{b, c, d, e}\end{array}$ & $\begin{array}{l}\text { Very uncertain } \\
\text { For maximum expiratory flow } \\
\text { after } 50 \% \text { of expired FVC } \\
\text { (change in mean \% predicted, } \\
\text { pre vs. post-bronchodilation } \\
\text { test) in adulthood ( } 17-20 \text { years } \\
\text { of age) among infants with vs. } \\
\text { without hospitalization for RSV at } \\
\text { age }<24 \text { months }\end{array}$ \\
\hline
\end{tabular}

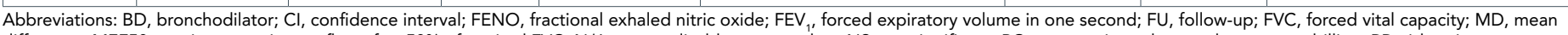
difference; MEF50, maximum expiratory flow after $50 \%$ of expired FVC; N/A, not applicable; no., number; NS, not significant; PC, prospective cohort; Ppb, parts per billion; RR, risk ratio; RSV, respiratory syncytial virus; RSV-H, respiratory syncytial virus hospitalization; vs., versus; wGA, weeks' gestational age; $y$, year(s)

a Absolute risk reductions were calculated when findings were statistically significant; NS denotes when findings were not statistically significant

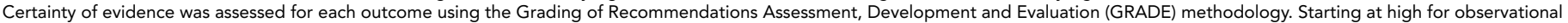

studies (for prognosis evidence) each outcome is rated as high, moderate, low or very low based on downgrading (if any) for one or more of the following domains:

${ }^{\mathrm{b}}$ Indirectness

' Imprecision

d Study limitations, including selective outcome reporting

e Two decrements (-2) due to very serious concerns for this domain 
Prematurity: One study enrolled premature (32-35 wGA) infants with or without hospitalization for RSV infection before 12 months of age to examine several long-term outcomes (32). Data were collected through telephone calls every four months and annual visits until the $6^{\text {th }}$ year of life. The authors analysed data both across the five years and only within the $6^{\text {th }}$ year. All findings offered low certainty evidence.

Across years two through six, associations were found between RSV-hospitalization and increased risk for parent or physician-reported simple wheeze, recurrent wheeze, severe wheeze and any/all wheeze (32). When examining the $6^{\text {th }}$ year only, there was little-to-no difference in parent or physician-reported simple wheeze, recurrent wheeze, severe wheeze and any/all wheeze (32). This study also compared groups for parent-reported asthma-associated medication use across years two through six. There were associations with increased risk from RSV-hospitalization for use of bronchodilators, inhaled corticosteroids, oral corticosteroids and leukotriene antagonists (32). Through lung function testing using spirometry, there was little-to-no difference in severe respiratory morbidity at six years of age between groups (32).

RSV without risk factors: Pooled data from two studies of children with versus without RSV-hospitalization at younger than 24 months of age found low certainty of an increase in self-reported asthma in adulthood $(27,28)$. Of note, there was no difference in physician-diagnosed asthma (considered more reliable than patient-reported asthma) (28), but certainty of evidence was very low for this outcome. An association was also found between RSV and lower pre-bronchodilation mean percent of predicted forced expiratory volume in one second $\left(F E V_{1}\right)$, but there was little-to-no difference in change in $\mathrm{FEV}_{1}$ from pre to post-bronchodilation $(27,28)$. The RSV was associated with lower predicted forced vital capacity, but not fractional exhaled nitric oxide $(27,28)$.

\section{Long-term outcome comparisons: Other data}

Supplement 11 contains single group data and pooled analyses (when appropriate). We did not conduct analyses for between-study comparator groups, since single studies that contributed to each long-term outcome were represented among within-study comparisons.

\section{Discussion}

\section{Summary of findings and limitations}

Few studies contributed data for within-study comparisons of outcomes of interest. There was moderate-to-low certainty that RSV is associated with a small increase in hospitalization and length of stay among moderate-to-late preterm (33-36 wGA) compared with term infants. There was moderate-to-low certainty evidence for no significant differences in hospitalizations between infants born at 29-32
wGA versus 33-36 wGA. Low certainty of evidence was found for a slight increase in mechanical ventilation among those born at 29-32 wGA versus 33-35 wGA and hospitalized for RSV prior to 12 months of age. Low certainty evidence was found for increased hospital length of stay among children younger than three years of age with versus without Down syndrome. There was low certainty evidence for increased wheezing and asthma medication use from two to six years of age among RSV-hospitalized versus non-hospitalized premature (32-35 wGA) infants, although there was little-to-no difference for these outcomes in the $6^{\text {th }}$ year of follow-up. Low certainty evidence was found for decreased lung function measurements before bronchodilation but changes in measurements after bronchodilation did not differ between groups. Very low certainty evidence was found for other long-term outcomes comparing different risk groups.

Single studies contributed data for most outcomes, where populations with rare conditions (e.g. cystic fibrosis) often represent small/under-powered sample sizes, precluding investigation of heterogeneity among studies for important population and RSV characteristics, or consistency in findings. The paucity of studies on clinical conditions other than prematurity is a limitation of the evidence base. We also did not find studies of premature infants born before 29-30 weeks gestation, or of children with chronic lung disease of prematurity or congenital heart disease, groups for whom prophylaxis is now recommended in the United States and in Canada $(2,23)$.

Retrospective study designs utilizing older data (i.e. pre-2014) were included, and may reflect different practices (e.g. prophylaxis, RSV-testing, standard of care) over time and across countries and settings. Detection of RSV infection may be impacted by variation in testing methods, including types of tests and indications for testing, and seasonal and geographic variability. Among tested individuals, the proportion of patients with viral or bacterial co-infections may be an important confounder in etiology of outcome severity. Lack of blinding of healthcare providers to risk status may influence rates of hospitalization and possibly other care parameters, particularly among children with known RSV risk factors.

\section{Comparison with other reviews}

A series of systematic reviews of publications from 1995 to 2015 found that RSV-hospitalization is associated with significant morbidity among children younger than 18 years old in Western countries (Canada, United States, Europe), particularly for young children with prematurity, chronic lung disease of prematurity and congenital heart disease (6-9). Whereas the current work focused on children younger than two years of age with a single risk factor, these reviews also included studies of children up to 18 years of age. Our review scope searched comparatively more recent publications (2014-2018) and covered a broader geographic area by including high-income (OECD) countries. 


\section{Future research}

Based on current evidence, there is a need for studies to focus on the burden of RSV disease among children with underlying chronic conditions, for some of which data on risk are contradictory or non-existing. Assessments of current RSV surveillance activities in Canada have identified data gaps for particular populations, including children with underlying medical conditions and those living in Indigenous, northern or remote communities (19). Gaps will need to be filled in preparation for monitoring of RSV vaccine effectiveness in the future.

\section{Conclusion}

Prematurity is associated with increased risk for RSVhospitalization in infancy and increased hospital length of stay, and may be associated with increased wheeze and asthmamedication use at up to six years of age. Down syndrome may be associated with longer hospital length of stay. We are very uncertain about evidence from other within-study comparisons. Very few studies included a comparison group.

\section{Authors' statement}

AW - Conceptualization, methodology, analysis, writing-original draft, review and editing

JP - Conceptualization, methodology, analysis, writing-review and editing

DLM - Conceptualization, methodology, analysis, writingreview and editing

SG - Analysis, writing-review and editing

BV - Analysis, writing-review and editing

MPD - Conceptualization, methodology, analysis

AS - Conceptualization, methodology, analysis, writing-review

and editing

MT - Conceptualization, methodology, writing-review and editing

LH - Conceptualization, methodology, writing-review and editing

\section{Competing interests}

AW, JP, SG, BV, MPD and LH report grants from the Public Health Agency of Canada during the conduct of the study.

\section{Acknowledgments}

The authors would like to thank Canada's National Advisory Committee on Immunization RSV Working Group (Drs. D Moore, J Papenburg, J Robinson, M Salvadori, W Vaudry, R Stirling, A Sinilaité, and R Pless) for their advice during initial study conception on strategic framing of the risk groups and for reviewing the search parameters. The authors thank Ms. L Gamble, librarian (Health Library of Health Canada and Public Health Agency of Canada), for conducting the literature search; Mr. S Duchesne-Belanger for assistance with title/abstract screening; and Dr. A Gates and Ms. S Rahman for assistance with data verification. The authors gratefully acknowledge Drs. K Backman and K Bønnelykke for providing study data.

\section{Funding}

This work was conducted for the National Advisory Committee for Immunization, under contract to the Public Health Agency of Canada (contract \#4600001536). The views of the funding body have not influenced the content of the review. The views expressed are those of the authors and do not necessarily represent those of the Public Health Agency of Canada. LH is supported by a Tier 1 Canada Research Chair in Knowledge Synthesis and Translation.

\section{Supplemental material}

These documents can be accessed on the Supplemental material file.

Supplement 1: Methods

Supplement 2: Search strategy

Supplement 3: Inclusion/exclusion criteria

Supplement 4: Methodological quality assessments

Supplement 5: Certainty of evidence assessments

Supplement 6: Characteristics of included studies

Supplement 7: Outcomes of included studies

Supplement 8: Single group proportions for short-term outcomes Supplement 9: Summary of evidence for short-term outcomesbetween-study

Supplement 10: Complications

Supplement 11: Single group proportions for long-term outcomes

\section{References}

1. Buchan SA, Chung $H$, Karnauchow T, McNally JD, Campitelli MA, Gubbay JB, Katz K, McGeer AJ, Richardson DC, Richardson SE, Simor A, Smieja M, Zahariadis G, Tran D, Crowcroft NS, Rosella LC, Kwong JC. Characteristics and outcomes of young children hospitalized with laboratory-confirmed influenza or respiratory syncytial virus in Ontario, Canada, 2009-2014. Pediatr Infect Dis J 2019;38(4):362-9. DOI PubMed

2. Robinson JL, Le Saux N; Canadian Paediatric Society, Infectious Diseases and Immunization Committee. Preventing hospitalizations for respiratory syncytial virus infection. Paediatr Child Health 2015;20(6):321-33. DOI PubMed

3. Barr R, Green CA, Sande CJ, Drysdale SB. Respiratory syncytial virus: diagnosis, prevention and management. Ther Adv Infect Dis 2019;6:2049936119865798. DOI PubMed 
4. Mitchell I, Defoy I, Grubb E. Burden of respiratory syncytial virus hospitalizations in Canada. Can Respir J 2017;2017:4521302. DOI PubMed

5. Pisesky A, Benchimol El, Wong CA, Hui C, Crowe M, Belair MA, Pojsupap S, Karnauchow T, O'Hearn K, Yasseen AS 3rd, McNally JD. Incidence of hospitalization for respiratory syncytial virus infection amongst children in Ontario, Canada: A Population-Based Study Using Validated Health Administrative Data. PLoS One 2016;11(3):e0150416. DOI PubMed

6. Figueras-Aloy J, Manzoni P, Paes B, Simões EA, Bont $L$, Checchia PA, Fauroux B, Carbonell-Estrany X. Defining the Risk and Associated Morbidity and Mortality of Severe Respiratory Syncytial Virus Infection Among Preterm Infants Without Chronic Lung Disease or Congenital Heart Disease. Infect Dis Ther 2016;5(4):417-52. DOI PubMed

7. Paes B, Fauroux B, Figueras-Aloy J, Bont L, Checchia PA, Simões EA, Manzoni P, Carbonell-Estrany X. Defining the risk and associated morbidity and mortality of severe respiratory syncytial virus infection among infants with chronic lung disease. Infect Dis Ther 2016;5(4):453-71. DOI PubMed

8. Checchia PA, Paes B, Bont L, Manzoni P, Simões EA, Fauroux B, Figueras-Aloy J, Carbonell-Estrany X. Defining the risk and associated morbidity and mortality of severe respiratory syncytial virus infection among infants with congenital heart disease. Infect Dis Ther 2017;6(1):37-56. DOI PubMed

9. Bont $L$, Checchia PA, Fauroux B, Figueras-Aloy J, Manzoni P, Paes B, Simões EA, Carbonell-Estrany $X$. Defining the epidemiology and burden of severe respiratory syncytial virus infection among infants and children in western countries. Infect Dis Ther 2016;5(3):271-98. DOI PubMed

10. Kristensen $K$, Hjuler T, Ravn H, Simões EA, Stensballe LG. Chronic diseases, chromosomal abnormalities, and congenital malformations as risk factors for respiratory syncytial virus hospitalization: a population-based cohort study. Clin Infect Dis 2012;54(6):810-7. DOI PubMed

11. Kua KP, Lee SW. Systematic review of the safety and efficacy of palivizumab among infants and young children with cystic fibrosis. Pharmacotherapy 2017;37(6):755-69. DOI PubMed

12. Manzoni $P$, Figueras-Aloy J, Simões EA, Checchia PA, Fauroux B, Bont L, Paes B, Carbonell-Estrany X. Defining the incidence and associated morbidity and mortality of severe respiratory syncytial virus infection among children with chronic diseases. Infect Dis Ther 2017;6(3):383-411. DOI PubMed

13. Banerji A, Panzov V, Young $M$, Robinson J, Lee $B$, Moraes T, Mamdani M, Giles BL, Jiang D, Bisson D, Dennis M, Morel J, Hall J, Hui C, Paes B, Mahony JB. Hospital admissions for lower respiratory tract infections among infants in the Canadian Arctic: a cohort study. CMAJ Open 2016;4(4):E615-22. DOI PubMed
14. Driscoll AJ, Arshad SH, Bont L, Brunwasser SM, Cherian T, Englund JA, Fell DB, Hammitt LL, Hartert TV, Innis BL, Karron RA, Langley GE, Mulholland EK, Munywoki PK, Nair H, Ortiz JR, Savitz DA, Scheltema NM, Simões EA, Smith PG, Were F, Zar HJ, Feikin DR. Does respiratory syncytial virus lower respiratory illness in early life cause recurrent wheeze of early childhood and asthma? Critical review of the evidence and guidance for future studies from a World Health Organization-sponsored meeting. Vaccine. 2020:2435-48. DOI

15. Jalink MB, Langley JM, Dodds L, Andreou P. Severe respiratory syncytial virus infection in preterm infants and later onset of asthma. Pediatr Infect Dis J 2019;38(11):1121-5. DOl PubMed

16. Shi T, Ooi Y, Zaw EM, Utjesanovic N, Campbell H, Cunningham S, Bont L, Nair H; RESCEU Investigators. Association between respiratory syncytial virus-associated acute lower respiratory infection in early life and recurrent wheeze and asthma in later childhood. J Infect Dis 2020;222 Suppl 7:S628-33. DOI PubMed

17. Andabaka T, Nickerson JW, Rojas-Reyes MX, Rueda JD, Bacic Vrca V, Barsic B. Monoclonal antibody for reducing the risk of respiratory syncytial virus infection in children. Cochrane Database Syst Rev 2013;4(4):CD006602. DOl PubMed

18. Robinson KA, Odelola OA, Saldanha IJ. Palivizumab for prophylaxis against respiratory syncytial virus infection in children with cystic fibrosis. Cochrane Database Syst Rev 2016;7(7):CD007743. DOI PubMed

19. Killikelly A, Shane A, Yeung MW, Tunis M, Bancej C, House A, Vaudry W, Moore D, Quach C. Gap analyses to assess Canadian readiness for respiratory syncytial virus vaccines: report from an expert retreat. Can Commun Dis Rep 2020;46(4):62-8. DOI PubMed

20. Higgins JP, Thomas J, Chandler J, Cumpston M, Li T, Page MJ, Welch VA, editors. Cochrane Handbook for Systematic Reviews of Interventions: Cochrane, version 6.2 (updated 2021-02; accessed 2021-02-24). www.training. cochrane.org/handbook

21. Iorio A, Spencer FA, Falavigna M, Alba C, Lang E, Burnand B, McGinn T, Hayden J, Williams K, Shea B, Wolff R, Kujpers T, Perel P, Vandvik PO, Glasziou P, Schunemann H, Guyatt G. Use of GRADE for assessment of evidence about prognosis: rating confidence in estimates of event rates in broad categories of patients. BMJ 2015;350:h870. DOI PubMed

22. Foroutan F, Guyatt G, Zuk V, Vandvik PO, Alba AC, Mustafa R, Vernooij R, Arevalo-Rodriguez I, Munn Z, Roshanov P, Riley R, Schandelmaier S, Kuijpers T, Siemieniuk R, Canelo-Aybar C, Schunemann H, lorio A. GRADE Guidelines 28: Use of GRADE for the assessment of evidence about prognostic factors: rating certainty in identification of groups of patients with different absolute risks. J Clin Epidemiol 2020;121:62-70. DOI PubMed 
23. American Academy of Pediatrics Committee on Infectious Diseases; American Academy of Pediatrics Bronchiolitis Guidelines Committee. Updated guidance for palivizumab prophylaxis among infants and young children at increased risk of hospitalization for respiratory syncytial virus infection. Pediatrics 2014;134(2):415-20. DOI PubMed

24. Murad MH, Montori VM, Walter SD, Guyatt GH. Estimating risk difference from relative association measures in meta-analysis can infrequently pose interpretational challenges. J Clin Epidemiol 2009;62(8):865-7.

DOI PubMed

25. Ambrose CS, Anderson EJ, Simões EA, Wu X, Elhefni $H$, Park CL, Sifakis F, Groothuis JR. Respiratory syncytial virus disease in preterm infants in the U.S. born at 32-35 weeks gestation not receiving immunoprophylaxis. Pediatr Infect Dis J 2014;33(6):576-82. DOI PubMed

26. Anderson EJ, Krilov LR, DeVincenzo JP, Checchia PA, Halasa N, Simões EA, Domachowske JB, Forbes ML, Pannaraj PS, McBride SJ, McLaurin KK, Kumar VR, Ambrose CS. SENTINEL1: an observational study of respiratory syncytial virus hospitalizations among U.S. infants born at 29 to 35 weeks' gestational age not receiving immunoprophylaxis. Am J Perinatol 2017;34(1):51-61. DOI PubMed

27. Backman K, Ollikainen H, Piippo-Savolainen E, Nuolivirta K, Korppi M. Asthma and lung function in adulthood after a viral wheezing episode in early childhood. Clin Exp Allergy 2018;48(2):138-46. DOl PubMed

28. Backman K, Piippo-Savolainen E, Ollikainen H, Koskela H, Korppi M. Adults face increased asthma risk after infant RSV bronchiolitis and reduced respiratory health-related quality of life after RSV pneumonia. Acta Paediatr 2014;103(8):850-5. DOI PubMed

29. Bjornson C, Chan P, Li A, Paes B, Lanctôt KL, Mitchell I. Palivizumab prophylaxis for respiratory syncytial virus in infants with cystic fibrosis: is there a need? Eur J Clin Microbiol Infect Dis 2018;37(6):1113-8. DOI PubMed

30. Blanken MO, Korsten $\mathrm{K}$, Achten NB, Tamminga S, Nibbelke EE, Sanders EA, Smit HA, Groenwold RH, Bont L; Dutch RSV Neonatal Network. Population-attributable risk of risk factors for recurrent wheezing in moderate preterm infants during the first year of life. Paediatr Perinat Epidemiol 2016;30(4):376-85. DOl PubMed

31. Bønnelykke K, Vissing NH, Sevelsted A, Johnston SL, Bisgaard $\mathrm{H}$. Association between respiratory infections in early life and later asthma is independent of virus type. J Allergy Clin Immunol 2015;136(1):81-86.e4. DOI PubMed

32. Carbonell-Estrany X, Pérez-Yarza EG, García LS, Guzmán Cabañas JM, Bòria EV, Atienza BB; IRIS (Infección Respiratoria Infantil por Virus Respiratorio Sincitial) Study Group. Long-term burden and respiratory effects of respiratory syncytial virus hospitalization in preterm infants-the SPRING study. PLoS One 2015;10(5):e0125422. DOI PubMed
33. Caserta MT, Qiu X, Tesini B, Wang L, Murphy A, Corbett A, Topham DJ, Falsey AR, Holden-Wiltse J, Walsh EE. Development of a Global Respiratory Severity Score for Respiratory Syncytial Virus Infection in Infants. J Infect Dis 2017;215(5):750-6. DOI PubMed

34. Chu PY, Hornik CP, Li JS, Campbell MJ, Hill KD. Respiratory syncytial virus hospitalisation trends in children with haemodynamically significant heart disease, 1997-2012. Cardiol Young 2017;27(1):16-25. DOI PubMed

35. Drummond D, Thumerelle C, Reix P, Fayon M, Epaud R, Clement A, Mahloul M, Habouria D, Delacourt C, Hadchouel A. Effectiveness of palivizumab in children with childhood interstitial lung disease: the French experience. Pediatr Pulmonol 2016;51(7):688-95. DOI PubMed

36. Farber HJ, Buckwold FJ, Lachman B, Simpson JS, Buck E, Arun M, Valadez AM, Ruiz T, Alonzo J, Henry A, Cos-Okpalla N, Nguyen K, Brendel W, Small J, Glomb WB. Observed effectiveness of palivizumab for 29-36-week gestation infants. Pediatrics 2016;138(2):e20160627. DOI PubMed

37. Fauroux B, Gouyon JB, Roze JC, Guillermet-Fromentin C, Glorieux I, Adamon L, Di Maio M, Anghelescu D, Miloradovich T, Escande B, Elleau C, Pinquier D. Respiratory morbidity of preterm infants of less than 33 weeks gestation without bronchopulmonary dysplasia: a 12-month follow-up of the CASTOR study cohort. Epidemiol Infect 2014;142(7):1362-74. DOI PubMed

38. Feldman AG, Sundaram SS, Beaty BL, Kempe A. Hospitalizations for Respiratory Syncytial Virus and Vaccine-Preventable Infections in the First 2 Years After Pediatric Liver Transplant. J Pediatr 2017;182:232-238.e1. DOI PubMed

39. Groves HE, Jenkins L, Macfarlane M, Reid A, Lynn F, Shields MD. Efficacy and long-term outcomes of palivizumab prophylaxis to prevent respiratory syncytial virus infection in infants with cystic fibrosis in Northern Ireland. Pediatr Pulmonol 2016;51(4):379-85. DOI PubMed

40. Hama I, Takahashi S, Nakamura T, Ito Y, Kawasaki K, Sago H. Risk of respiratory syncytial virus infection in infants with congenital cystic lung disease. Pediatr Int 2015;57(2):253-7. DOI PubMed

41. Hatanaka M, Miyamura T, Koh K, Taga T, Tawa A, Hasegawa D, Kajihara R, Adachi S, Ishii E, Tomizawa D. Respiratory syncytial virus infection in infants with acute leukemia: a retrospective survey of the Japanese Pediatric Leukemia/Lymphoma Study Group. Int J Hematol 2015;102(6):697-701. DOI PubMed

42. Helfrich AM, Nylund CM, Eberly MD, Eide MB, Stagliano DR. Healthy Late-preterm infants born 33-36+6 weeks gestational age have higher risk for respiratory syncytial virus hospitalization. Early Hum Dev 2015;91(9):541-6. DOl PubMed 
43. Korsten K, Blanken MO, Nibbelke EE, Moons KG, Bont L; Dutch RSV Neonatal Network. Prediction model of RSV-hospitalization in late preterm infants: an update and validation study. Early Hum Dev 2016;95:35-40. DOI PubMed

44. Luchsinger V, Ampuero S, Palomino MA, Chnaiderman J, Levican J, Gaggero A, Larrañaga CE. Comparison of virological profiles of respiratory syncytial virus and rhinovirus in acute lower tract respiratory infections in very young Chilean infants, according to their clinical outcome. J Clin Virol 2014;61(1):138-44. DOl PubMed

45. McLaurin KK, Farr AM, Wade SW, Diakun DR, Stewart DL. Respiratory syncytial virus hospitalization outcomes and costs of full-term and preterm infants. J Perinatol 2016;36(11):990-6. DOI PubMed

46. O'Brien $\mathrm{KL}$, Chandran A, Weatherholtz R, Jafri HS, Griffin MP, Bellamy T, Millar EV, Jensen KM, Harris BS, Reid R, Moulton LH, Losonsky GA, Karron RA, Santosham M; Respiratory Syncytial Virus (RSV) Prevention study group. Efficacy of motavizumab for the prevention of respiratory syncytial virus disease in healthy Native American infants: a phase 3 randomised double-blind placebo-controlled trial. Lancet Infect Dis 2015;15(12):1398-408. DOI PubMed

47. Rajah B, Sánchez PJ, Garcia-Maurino C, Leber A, Ramilo O, Mejias A. Impact of the Updated Guidance for Palivizumab Prophylaxis against Respiratory Syncytial Virus Infection: A Single Center Experience. J Pediatr 2017;181:183-188.e1. DOI PubMed

48. Ryan VM, Langley JM, Dodds L, Andreou P. Estimating respiratory syncytial virus-associated hospitalization in the first year of life among infants born at 32-35 weeks of gestation. Pediatr Infect Dis J 2016;35(8):851-5.

DOI PubMed
49. Sadreameli SC, Reller ME, Bundy DG, Casella JF, Strouse JJ. Respiratory syncytial virus and seasonal influenza cause similar illnesses in children with sickle cell disease.

Pediatr Blood Cancer 2014;61(5):875-8. DOI PubMed

50. Stagliano DR, Nylund CM, Eide MB, Eberly MD. Children with Down syndrome are high-risk for severe respiratory syncytial virus disease. J Pediatr 2015;166(3):703-9.e2. DOl PubMed

51. Straňák Z, Saliba E, Kosma P, Posfay-Barbe K, Yunis K, Farstad T, Unnebrink K, van Wyk J, Wegzyn C, Notario G, Kalus S, Campbell FJ. Predictors of RSV LRTI hospitalization in infants born at 33 to 35 weeks gestational age: a large multinational study (PONI). PLoS One 2016;11(6):e0157446. DOI PubMed

52. Zomer-Kooijker K, Uiterwaal CS, van der Gugten AC, Wilbrink B, Bont LJ, van der Ent CK. Decreased lung function precedes severe respiratory syncytial virus infection and post-respiratory syncytial virus wheeze in term infants. Eur Respir J 2014;44(3):666-74. DOI PubMed

53. Franklin JA, Anderson EJ, Wu X, Ambrose CS, Simões EA. Insurance status and the risk of severe respiratory syncytial virus disease in United States preterm infants born at 32-35 weeks gestational age. Open Forum Infect Dis 2016;3(3):ofw163. DOI PubMed

54. Simões EA, Anderson EJ, Wu X, Ambrose CS. Effects of chronologic age and young child exposure on respiratory syncytial virus disease among US preterm infants born at 32 to 35 weeks gestation. PLoS One 2016;11(11):e0166226. DOl PubMed 